\title{
Les paysages de l'écologie : traversée critique des modèles paysagers, de la fiction verte à l'écopoétique
}

\author{
The landscapes of ecology: a critical journey throught landscape \\ models, from green fiction to ecopoetics
}

\author{
Anaïs Belchun ${ }^{1}$ \\ ${ }^{1}$ LARA-SEPPIA, Université Toulouse - Jean Jaurès (contact@anaisbelchun.com)
}

\begin{abstract}
RÉSUMÉ. L'écologie étudie les relations entre les êtres vivants et leur milieu de vie. Une approche culturelle des problématiques écologiques interroge les relations sensibles et signifiantes que nous, humains, établissons avec nos milieux de vie. Le paysage nous permet d'aborder ces questions de manière sensible. La notion même de paysage est remise en question par l'approche écologique. Je propose cependant de reconsidérer l'intérêt d'une approche paysagère, à condition d'élargir le sens de cette notion, en identifiant différents modèles paysagers, et en les interrogeant au regard de l'écologie. Cette proposition se fonde sur une distinction entre la fiction verte et l'écopoétique. La fiction verte est constituée de représentations issues d'une approche superficielle de l'écologie : le paysage-décor, le paysage-émotion, le paysage-spectacle et le paysage-catastrophe. Les pistes qui s'avancent vers une écopoétique paysagère expriment une approche plus profonde, à travers les modèles du paysage-système, du paysage-cosmos, du paysage-vivant et du paysage-milieu de vie. Une traversée critique de ces différents modèles paysagers nous permettra de saisir les enjeux écologique du paysage, qui reflète notre vision du monde et notre manière d'interagir avec notre milieu de vie commun : la Terre.
\end{abstract}

ABSTRACT. Ecology studies the relationships between living beings and their environment. A cultural approach of ecological issues questions the sensitive and significant relationships that we humans establish with our living environments. Landscape allows us to approach these issues in a sensitive way. The very notion of landscape is challenged by the ecological approach. However, I propose to reconsider the value of a landscape approach (provided that the meaning of this concept is broadened), by identifying different landscape models, and by questioning them in terms of ecology. This proposal is based on a distinction between green fiction and ecopoetics. Green fiction is made of representations from a superficial approach of ecology : the scenery-landscape, the emotion-landscape, the spectacle-landscape and the disaster-landscape. Ways to ecopoetics express a deeper approach, through the models of the system-landscape, the cosmos-landscape, the living-landscape and the living environment-landscape. A critical journey through these different landscape models will allow us to grasp the ecological challenges of landscape, which reflects our vision of the world and our ways of interact with our common environment : the Earth.

MOTS-CLÉS. paysage, écologie, écopoétique, environnement, décor, émotion, spectacle, catastrophe, système, cosmos, vivant, milieu.

KEYWORDS. landscape, ecology, ecopoetics, environment, scenery, emotion, spectacle, disaster, system, cosmos, living. 


\section{Introduction. Approches écologiques du paysage : quels modèles ?}

L'écologie devient un sujet de réflexion de plus en plus important de nos jours, interrogeant nos rapports à la nature, dans tous les domaines de notre vie. Le paysage nous permet d'aborder ces questions de manière sensible, mais la notion même de paysage est aussi remise en question par l'écologie. Je propose ici d'explorer la manière dont on peut reconsidérer différents modèles paysagers, au regard de l'écologie.

Effectuant des recherches sur la manière dont une pensée de l'écologie peut être mise en œuvre dans les arts du paysage, je me suis d'abord interrogée sur ce que serait une pensée de l'écologie. J'ai découvert de nombreuses pistes, parcourant différents domaines de pensée: l'écologie scientifique et la pensée systémique, l'écologie politique et philosophique, des approches théoriques dans le champ des sciences humaines (avec la géographie, l'anthropologie, la psychologie, entre autres), ainsi que des approches plus pragmatiques dans les domaines du paysagisme, de l'agriculture, de l'aménagement du territoire, etc.

Au-delà de ces distinctions, ces pistes peuvent converger vers une approche transdisciplinaire de l'écologie, qui me semble marquer l'émergence d'une vision du monde écologique. On peut en fait repérer dans les discours et les représentations associés à une pensée de l'écologie deux axes suivant globalement la distinction établie par Arne Naess entre «écologie superficielle et « écologie profonde ${ }^{1}$. Ils se distinguent par une vision du monde, un positionnement éthique, des esthétiques et des pratiques différentes.

Le premier axe est fondé sur une approche environnementale, anthropocentrée et utilitariste de la nature, associé au modèle du développement durable. Je nomme «fiction verte » l'ensemble des discours et représentations qui en émerge. Ces représentations sont souvent fondées sur une image idéalisée de la Nature (définie en opposition avec la Culture). À l'extrême, on trouve des images simplistes faites pour produire un «effet-écologie», dans lesquelles la nature est réduite à la végétation, voire à la couleur verte - d'où le nom de fiction verte. Cette vision est finalement peu représentative d'une pensée de l'écologie (quand ce n'est pas juste un moyen de détournement idéologique, comme dans le cas du greenwashing $)^{2}$.

Le second axe est celui d'une pensée plus complexe, fondée sur la mise en relation entre l'écologie scientifique, les pensées de l'écologie issues des sciences humaines, et diverses pratiques (agricoles, paysagères, artistiques...). Cette pensée s'articule autour de deux problématiques: comment habiter la Terre ? et comment vivre dans la communauté du Vivant ? Je propose le terme d'écopoétique (issu du domaine littéraire) pour désigner cette vision du monde, car elle présente aussi une dimension plus sensible, exprimée dans une relation au monde esthétique ou poétique, ou encore dans des pratiques artistiques.

Je me suis ensuite interrogée sur ce qu'est le paysage. Il existe de nombreuses réponses à cette question, selon qu'on s'adresse à un peintre ou un géographe, un agriculteur, un philosophe ou un paysagiste, etc. Pour s'y retrouver dans la grande diversité d'approches possibles, on peut les classer selon des distinctions disciplinaires ou des postures professionnelles. C'est ainsi que Jean-Marc Besse présente cinq portes - ou cinq définitions du paysage : comme représentation (culturelle et

\footnotetext{
${ }^{1}$ Naess, A. « Le mouvement d'écologie superficielle et le mouvement d'écologie profonde de longue portée. Une présentation ".
}

2 Belchun, Anaïs. "Écofictions paysagères : la fiction verte et ses alternatives ", Séminaire " Images des lieux, dispositifs esthétiques et enjeux politiques ", Université de Toulouse - Jean Jaurès, Toulouse, 2015. Hal-01427495. 
sociale), comme territoire (fabriqué et habité par les sociétés humaines), comme écoumène (système naturel et culturel constituant l'environnement humain), comme expérience sensible, ou encore comme projet ${ }^{3}$.

J'essaie de prendre en compte toutes ces différentes approches du paysage, en cherchant comment elles peuvent être mises en relation avec l'écologie. Dans un premier temps, j'ai cherché à constituer deux modèles paysagers, en rapport avec les deux visions de l'écologie. Mais les représentations paysagères que j'étudiais se sont révélées trop complexes et variées pour se limiter à ces deux modèles. Je me suis alors inspirée d'un article de Roger Cavailles ${ }^{4}$, dans lequel il propose trois modèles paysagers, associés à différentes approches de l'écologie. Passons le fait qu'il commence par évacuer l'écologie scientifique, au prétexte qu'elle se soucie d'environnement et non de paysage (ce qui peut être discuté, et nous en reparlerons plus tard). Il présente d'abord le modèle du paysage-décor, associé à une approche cartésienne et anthropocentrée de l'écologie : le paysage est perçu comme objet, ou ensemble d'objets dans l'espace, constituant un décor pour le sujet qui le contemple. Il oppose ensuite à ce modèle celui du paysage-Nature, associé à une approche romantique et éco-centrée de l'écologie : le paysage est perçu comme Sujet, en présence duquel on éprouve une émotion. Il juge cependant le modèle du paysage-décor trop matérialiste, et celui du paysage-Nature trop irrationnel. Il propose donc une alternative : celle du paysage-lieu de vie, abordée selon une éthique de la responsabilité. Le paysage est alors considéré comme une notion concrète et locale, dans ses dimensions à la fois matérielle et culturelle. Ce modèle remet en question la distinction entre nature et culture, comme le font de plus en plus de penseurs, philosophes, anthropologues ou géographes (comme Philippe Descola ${ }^{5}$, Augustin Berque ${ }^{6}$ ou Tim Ingold $^{7}$ ).

À partir de ces exemples, j'ai conçu un essai de typologie de plusieurs modèles paysagers. Ils se déploient entre le paysage-décor et le paysage-milieu de vie, et s'organisent autour de deux axes principaux qui sont la fiction verte et l'écopoétique. Comme modèles de la fiction verte paysagère, je vais vous présenter ma version du paysage-décor, mais aussi le paysage-émotion, le paysagespectacle et le paysage-catastrophe. Comme pistes vers une écopoétique paysagère, j'explorerai les modèles du paysage-système, du paysage-cosmos, du paysage-vivant, et enfin du paysage-milieu de vie.

Cette modélisation pourra sembler un peu caricaturale : précisons qu'il s'agit seulement d'un outil conceptuel, par nécessité moins complexe que la réalité. Nos représentations paysagères se constituent en fait de ce qui traverse ces différents modèles, mais aussi de ce qui se tisse entre eux, et de ce qui leur échappe... Je vous propose maintenant une traversée rapide de ces modèles paysagers, en développant un regard critique pour comprendre comment chacun d'eux se rattache soit à la fiction verte, soit à l'écopoétique.

\footnotetext{
${ }^{3}$ Besse, J-M. « Les cinq portes du paysage, essai d'une cartographie des problématiques paysagères contemporaines ", dans Le goût du monde, exercices de paysage.

${ }^{4}$ Cavailles, R. « Le philosophe, le paysage et l'écologie » dans Le paysage : 13e colloque d'Albi.

${ }^{5}$ Descola, P. Par-delà nature et culture.

${ }^{6}$ Berque, A. Poétique de la terre.

${ }^{7}$ Ingold T. Marcher avec les dragons.
} 


\section{MODÈLES DE LA FICTION VERTE PAYSAGÈRE}

\section{Le paysage - décor}

Le premier modèle que j'associe à la fiction verte paysagère est celui du paysag-décor. Il s'agit d'une perception du paysage caractéristique de notre culture occidentale, issue d'une ontologie naturaliste (d'après la définition de Philippe Descola ${ }^{8}$ ). La Nature étant définie par Descartes comme «l'ensemble des objets étalés dans l'espace », elle est perçue à travers ses caractéristiques physiques, concrètes et mesurables.

La notion de paysage se réfère à l'aspect visible de cette nature, tel qu'il se présente au regard d'un sujet (c'est pourquoi Roger Cavailles ${ }^{9}$ définissait le paysage-décor comme objet). Cela correspond à la première définition du paysage que l'on trouve dans le dictionnaire Larousse : « étendue spatiale, naturelle ou transformée par l'homme, qui présente une certaine identité visuelle ou fonctionnelle ».

Le paysage est donc perçu comme « un ensemble d'objets étalés dans l'espace », constituant le décor dans lequel évolue un sujet humain, selon une appréciation uniquement visuelle ou fonctionnelle. Le Larousse nous en donne aussi une deuxième définition : " vue d'ensemble qu'on a d'un point donné ». Ceci renvoie à une posture esthétique, qui selon Allen Carson, consiste à « apprécier l'environnement comme si c'était une peinture de paysage ${ }^{10}$. Voici quelques exemples de représentations que j'associe au modèle du paysage-décor.

Ce modèle paysager est apparu dans la peinture occidentale à la Renaissance, d'abord comme image de fond cadré par une fenêtre, comme dans le tableau de Jan Van Eyck La vierge du chancelier Rolin (illustration 1). Son rôle de décor est ici explicite, on voit une séparation nette entre les personnages à l'intérieur (sujet principal du tableau), et le paysage à l'extérieur.

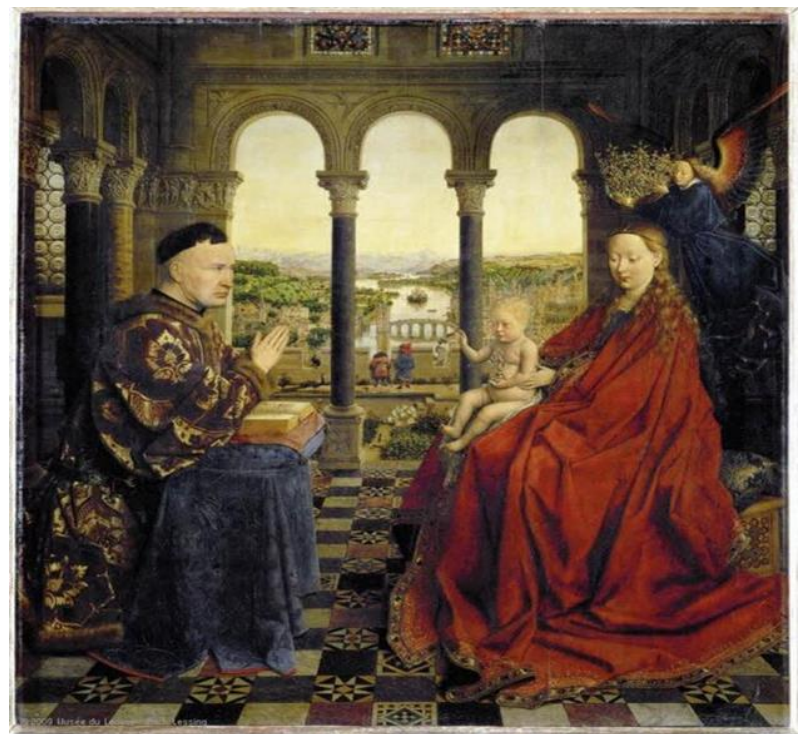

Illustration 2 : Jan Van Eyck « La Vierge du Chancelier Rolin » (Pays-Bas, 1435)

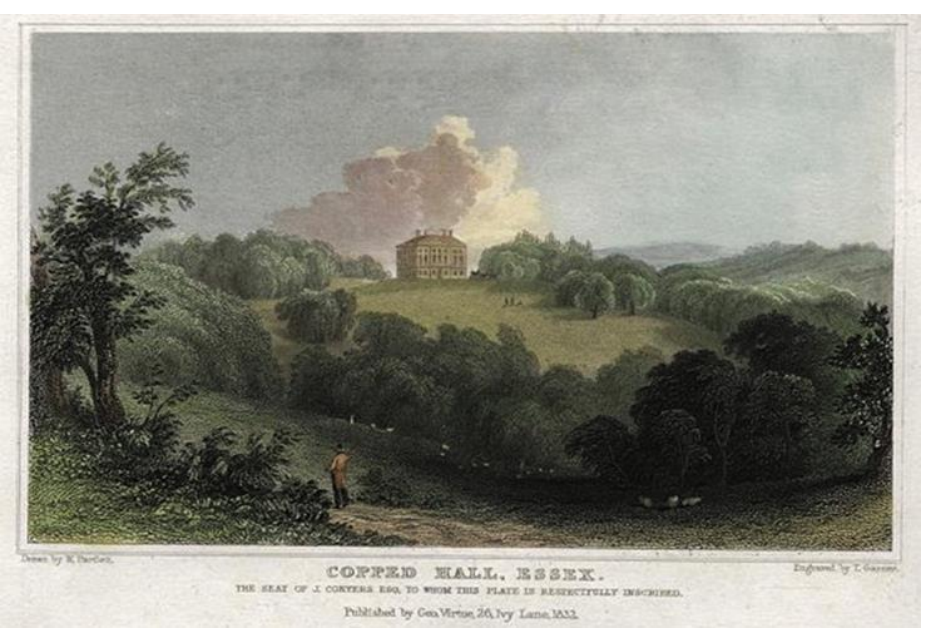

IIlustration 1 : George Virtue, Copped Hall, Essex (Angleterre, 1832)

\footnotetext{
${ }^{8}$ Descola, P. Par-delà nature et culture.

${ }^{9}$ Cavailles, R. « Le Philosophe, Le Paysage et l'écologie ».

${ }^{10}$ Carlson, A. «L'appréciation esthétique de l'environnement naturel», dans : Afeissa, Hicham-Stéphane (dir.), Esthétique de l'environnement.
} 
Puis le paysage a progressivement envahi le cadre, jusqu'à être jugé digne d'être représenté pour lui-même. On assiste alors à l'apparition de l'esthétique pittoresque, qui désigne un point de vue particulièrement intéressant, ou un objet «digne d'être peint » (illustration 2). Contrairement à ce qu'on pourrait croire, la séparation entre le paysage (la nature) et l'homme (la culture) est alors amplifiée : le paysage n'est plus qu'un objet (un tableau ou une photographie) posé sous le regard du sujet, qui le contemple de l'extérieur.

Ce modèle ne s'est pas diffusé que dans les représentations picturales, mais aussi dans les aménagements paysagers. Un exemple caractéristique est celui des jardins du Château de Versailles (illustration 3). Conçu selon un modèle géométrique, il remplit une double fonction en tant qu'espace aménagé pour ses usagers : une fonction utilitaire, consistant à orienter les déplacements, et une fonction esthétique, consistant à produire un point de vue agréable mais aussi à orienter le regard.

Cette approche du paysage est encore majoritaire de nos jours. On la retrouve par exemple dans les villes du monde entier, dans l'aménagement des parcs ou des « espaces verts ( (illustration 4). Le rôle de l'aménagement paysager est ici aussi limité à une fonction utilitaire et d'agrément. On constate une séparation nette entre les zones d'usage (pelouses et chemins, d'ailleurs pré-formatés pour marcher ici ou s'assoir là) et les zones de décor (arbres et massifs fleuris). Le concept d'espace vert, qui désigne en urbanisme « un espace d'agrément végétalisé », est caractéristique de la fiction verte, la nature étant réduite à la végétation et à la couleur verte.

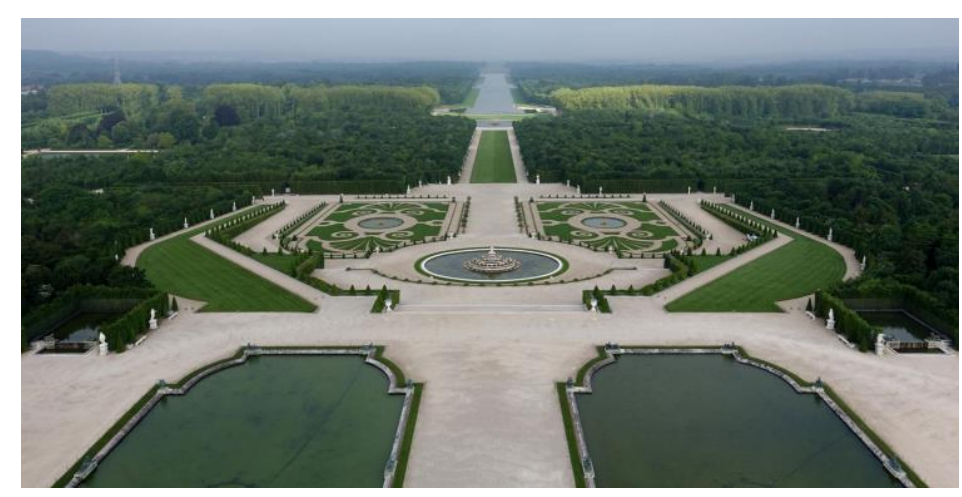

Illustration 3 : Jardins de Versailles, conçus par André Le Nôtre (France, XVIle siècle)

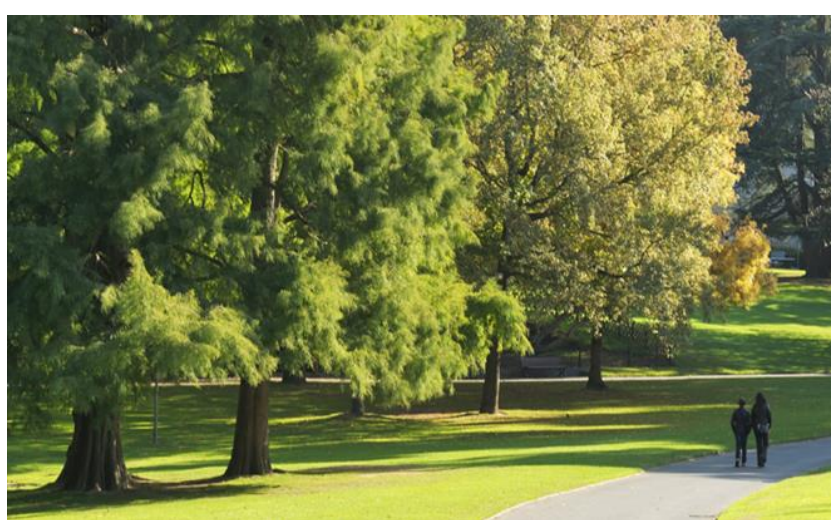

Illustration 4 : Parc de Procé (Nantes, France, XIXe siècle)

En résumé, le modèle du paysage-décor se fonde sur une vision du monde naturaliste, cartésienne et anthropocentrée, et est mis en œuvre selon une approche mécaniste et fonctionnaliste, ou même utilitariste. Le paysage est réduit à un espace ou une image de fond, jouant un rôle utilitaire et esthétique au profit d'un sujet humain.

Quand ce modèle paysager est utilisé en relation avec un discours sur l'écologie, celui-ci se révèle souvent fondé sur une approche superficielle de l'écologie. Par exemple, des images pittoresques d'un paysage de « nature sauvage » peuvent être utilisées pour promouvoir des produits ou services dits «verts » (mais le sont-ils vraiment ?). La réalisation d'espaces verts dans une ville peut être mise en avant en tant que politique de développement durable (mais l'écologie se limite-telle à des mesures de $\mathrm{CO}^{2}$ ?).

Le paysage-décor peut donc servir la fiction verte, mais on peine à voir ses relations avec une pensée ou une sensibilité véritablement écologiste. 


\section{Le paysage - émotion}

En réaction à cette approche cartésienne et fonctionnaliste de la nature, que l'on peut juger contraire à une sensibilité écologique, voire même déshumanisante, un autre modèle de représentation de la nature est souvent avancé : celui du romantisme. Bien que l'on soit toujours dans le cadre d'une vision du monde naturaliste, on s'éloigne de l'approche matérialiste. Cette approche valorise l'émotion, comme voie d'accès à une vie en harmonie avec la Nature.

Pour les poètes romantiques du XIX ${ }^{\mathrm{e}}$ siècle, la valeur de la Nature est d'abord un concept symbolique : elle représente l'incarnation de Dieu. Dans sa dimension matérielle et spatiale, elle constitue un lieu de refuge (loin de la civilisation urbaine), de recueillement et de contemplation. Mais la nature est avant tout considérée comme la source ou le reflet des émotions humaines.

Les représentations paysagères romantiques exemplifient parfaitement cette approche. Soit la nature perçue par l'artiste provoque en lui une émotion; soit il utilise une représentation paysagère pour traduire un état d'âme. Dans les deux cas, c'est une émotion qu'il tente de transmettre au spectateur à travers la peinture de paysage. C'est pourquoi je nomme ce modèle le paysage-émotion.

Prenons par exemple le tableau de Caspar Friedrich,L'abbaye dans une forêt de chênes (illustration 5). Dans cette scène d'enterrement, tous les éléments du paysage évoquent la mort et suggère la tristesse et la mélancolie : l'église en ruine, le cimetière sous la neige, les arbres sans feuilles, le soleil couchant. Il semblerait que l'on assiste ici à une ré-unification de l'homme avec la nature, par l'harmonie présentée entre le paysage et les émotions. Mais l'on pourrait avancer au contraire que la nature véritable est ici totalement nié, puisque qu'on ne montre que des éléments symboliques, et que les représentations paysagères n'évoquent en fait que les tourments de l'âme

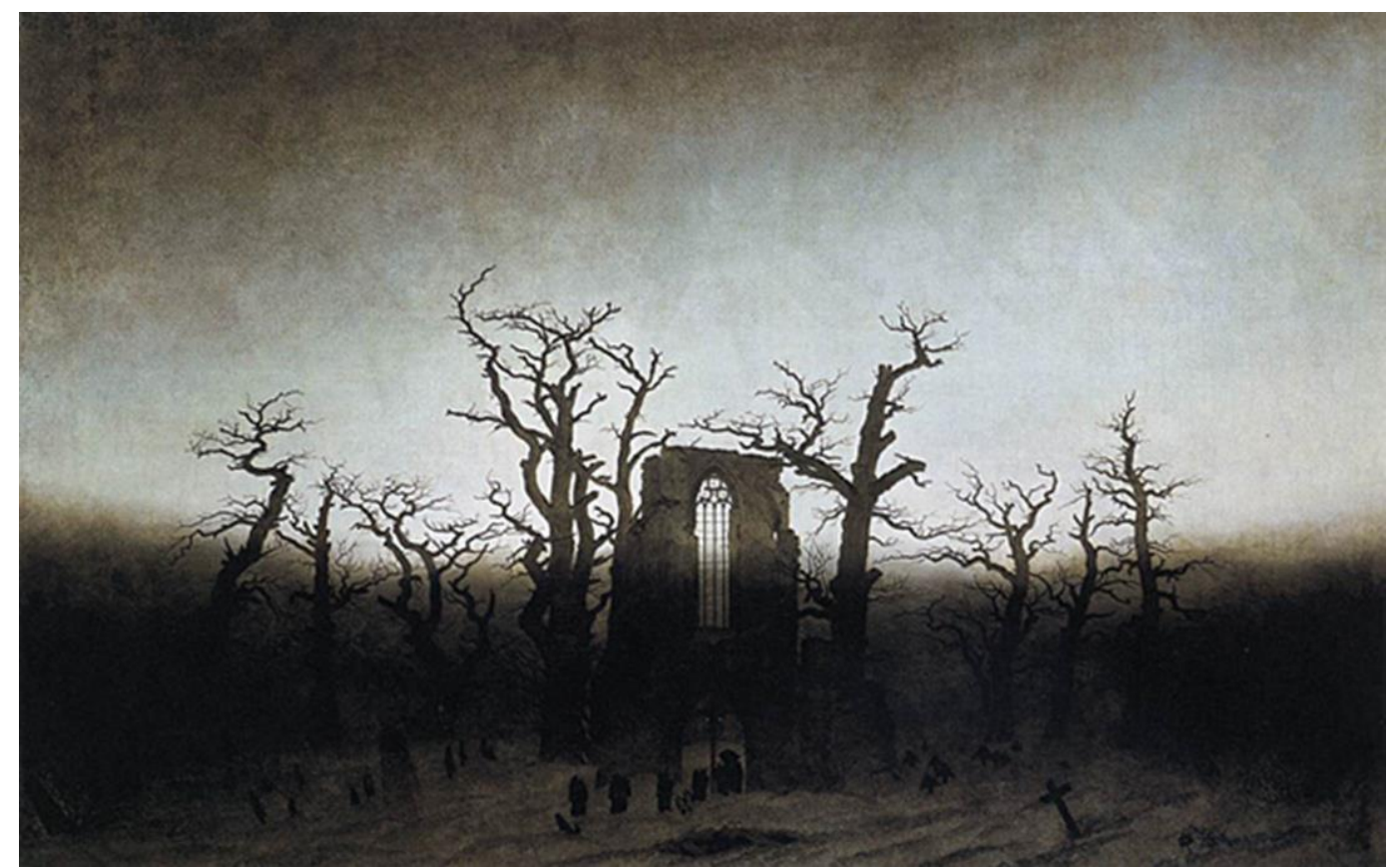

Illustration 5 : Caspar Friedrich, L'abbaye dans une forêt de chênes (Allemagne, 1810)

humaine. Le paysage-émotion révèle une approche totalement anthropocentrée.

Une autre esthétique propre au paysage romantique est souvent mise en avant comme modèle de valorisation de la Nature : le sublime. En voici un exemple, avec les Pêcheurs en mer de Turner (illustration 6). Tous les éléments du paysage (les mouvements des vagues et des nuages, les couleurs du ciel et de la mer, l'éclairage contrasté) concourent à créer un effet dramatique, 
provoquant un sentiment d'effroi admiratif face au contraste entre la puissance de la nature et la vulnérabilité des pêcheurs. On peut penser que cette nature grandiose, sauvage et belle, qui domine l'être humain, pourrait être un modèle pour une éthique écologiste. Mais le sentiment d'admiration provoqué par ce tableau est-il vraiment dirigé vers la nature, ou vers les courageux pêcheurs qui osent l'affronter...?

Le sentiment esthétique du sublime est en fait paradoxal, à plusieurs niveaux. D'abord, il associe l'effroi et l'admiration. Ce qui nous permet de s'abandonner à ce sentiment d'admiration, c'est le fait que nous ne soyons pas en situation de danger (en contemplant une peinture...). La puissance de

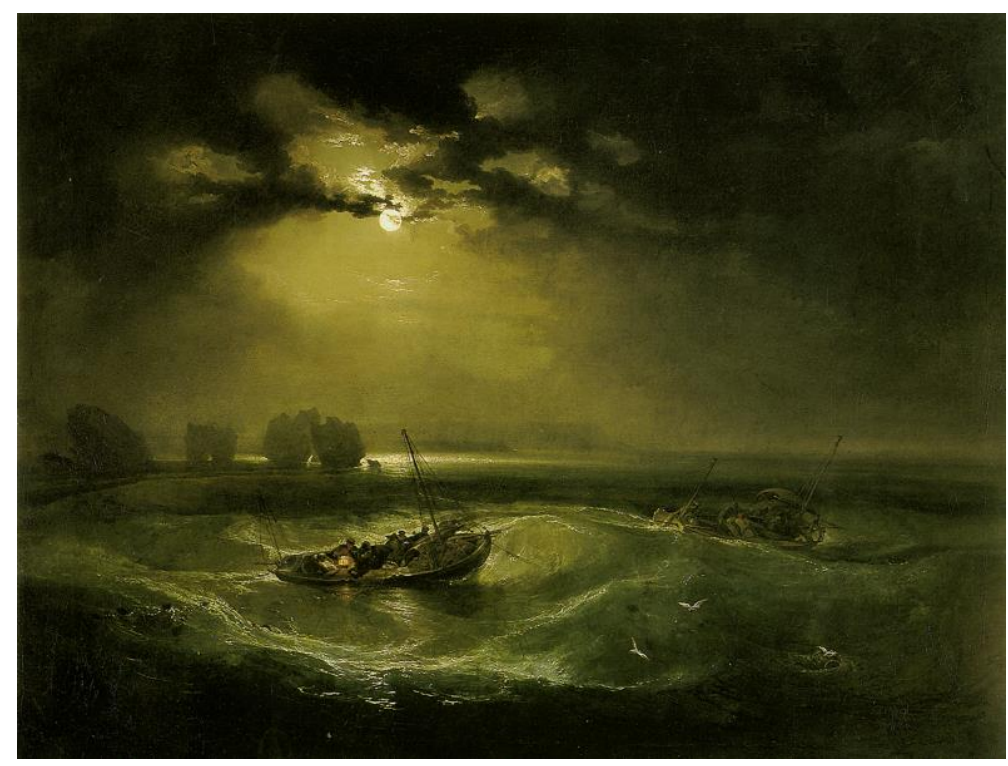

Illustration 6 : William Turner, Pêcheurs en mer.

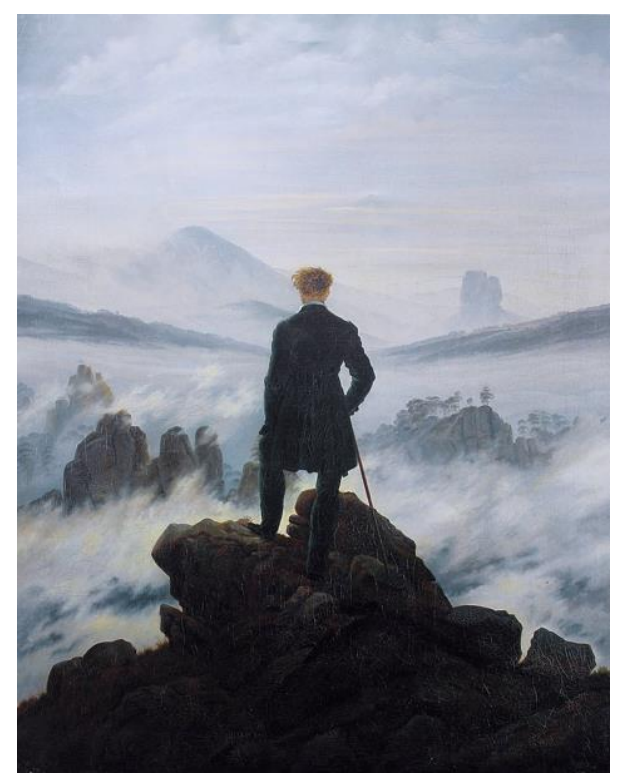

Illustration 7 : Caspar Friedrich, Le voyageur au-dessus de la mer de nuages (Allemagne, 1818)

la nature est donc compensée par la distance d'où elle est contemplée.

Cette posture est révélée par exemple dans un autre tableau de Friedrich : Le voyageur au-dessus de la mer de nuages (illustration 7). Les interprétations de ce tableau se focalisent souvent sur l'intériorité humaine : que pense, que ressent ou que symbolise ce personnage ? Ce qui est cohérent avec l'intention de Friedrich, qui disait que « le peintre ne doit pas peindre seulement ce qu'il voit en face de lui, mais aussi ce qu'il voit en lui. » Mais si l'on s'intéresse plutôt au paysage représenté, il nous révèle une autre face de la relation romantique entre l'Homme et la Nature. Celle-ci se présente ici sous l'apparence de pics rocheux couverts de brume: c'est-à-dire un espace sauvage, inhospitalier et incompréhensible. L'Homme étant dans une position de domination, extérieur à cette nature qu'il contemple de haut.

Contrairement aux apparences, l'esthétique romantique du paysage-émotion préserve donc la séparation ontologique entre Nature et Culture, ainsi qu'une sensibilité anthropocentrée. Car la vision de la nature qui est valorisée est celle d'une nature totalement fantasmée. Soit le paysage est le reflet des émotions humaines, auquel cas la nature réelle disparaît des représentations au profit de l'intériorité humaine. Soit le paysage représente une nature sauvage (presque caricaturale), que l'homme combat ou domine de haut - ce qui suggère une opposition entre les deux, toujours au profit de l'humain.

En résumé, le modèle du paysage-émotion perpétue une vision du monde naturaliste et anthropocentrée, valorisant les émotions et la grandeur de l'âme humaine face à une nature fantasmée. La fiction verte tourne à plein régime, et même les écologistes s'en réclame... mais nous 
sommes pourtant bien loin des savoirs issus de l'écologie scientifique comme des concepts de l'écologie philosophique et culturelle.

\section{Le paysage - spectacle}

Dans les représentations contemporaines, les deux modèles du paysage-décor et du paysageémotion sont souvent combinées pour montrer la beauté de la nature, parfois dans l'objectif explicite de promouvoir une prise de conscience écologique. L'origine de cette approche se trouve peut-être dans la photographie américaine du début du vingtième siècle, par exemple dans les photographies d'Ansel Adams (illustration 8).

On retrouve dans ses images le sentiment du sublime hérité de l'esthétique romantique, mais avec une approche qui se veut plus rationnelle : la subjectivité revendiquée de la peinture romantique est remplacée par la supposée objectivité de la technique photographique. L'intention n'est plus d'exprimer des sentiments humains mais de présenter et de mettre en valeur la beauté de la nature pour elle-même. Ansel Adams souhaitait valoriser le «patrimoine naturel » que constituent les paysages américains, et joua ainsi un rôle dans la création de plusieurs parcs nationaux.

On constate aussi un retour de l'esthétique pittoresque, propre au paysage-décor, avec ses paysages soigneusement cadrés depuis un point de vue privilégié. L'émotion n'est toutefois pas absente de ce modèle paysager : le pittoresque n'est plus destiné à être un simple agrément, mais vise à provoquer une émotion chez le spectateur. Le paysage est devenu un spectacle à contempler, servant à diffuser un message : c'est pourquoi je propose de nommer ce modèle paysager le

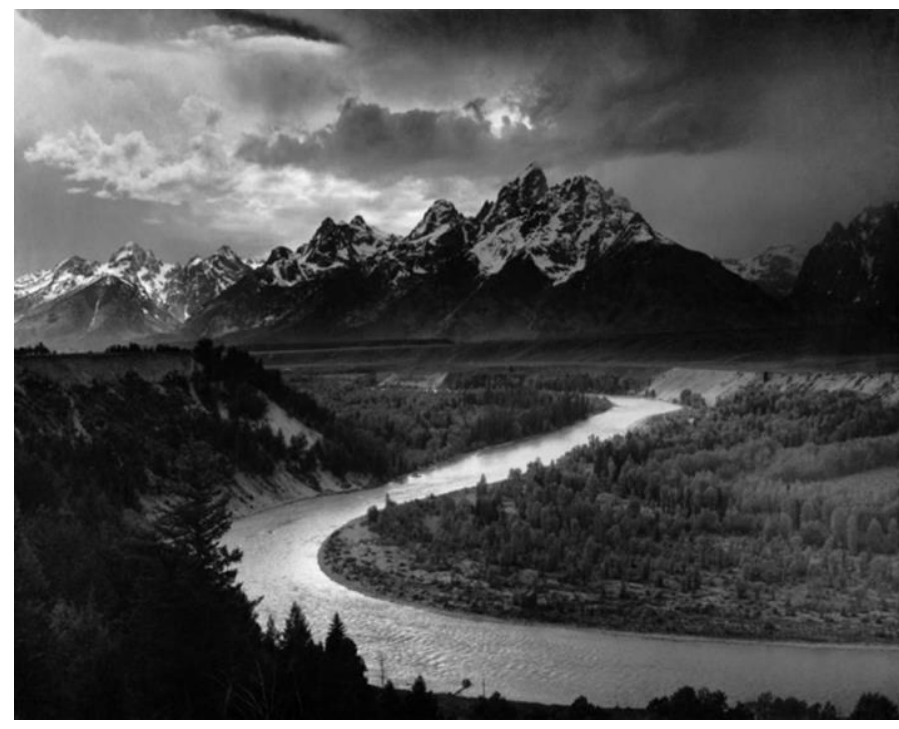

Illustration 8 : Ansel Adams, The Tetons and the Snake River (USA, 1942)

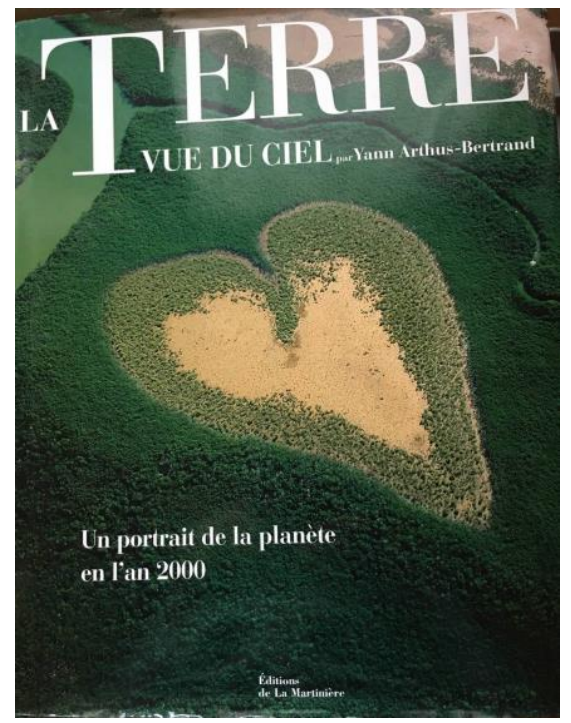

Illustration 9 : Yann Arthus Bertrand, Couverture de La Terre vue du ciel

(France, 1999)

paysage-spectacle.

Un exemple emblématique est fourni par les photographies de La Terre vue du ciel de Yann Arthus-Bertrand (illustration 9), livre réalisé dans l'intention de «témoigner de la beauté du monde et tenter de protéger la Terre ». Il a ensuite utilisé le même principe pour réaliser dix ans après le film Home. Mais on remarquera que (selon les termes d'Estelle Bayon) « l'image aérienne reste une fascination de surface (...): la Terre n'est qu'un prétexte à faire de belles images ». Le discours moralisateur du film est ainsi neutralisé par cette esthétisation graphique. D'ailleurs, ces œuvres ont été saluées pour leur action de sensibilisation à l'environnement... mais elles ont aussi été très critiquées par des militants écologistes, qui dénoncent la contradiction entre le message écologiste 
revendiqué et la vision du monde véhiculée par ces images. La posture extérieure et dominante du spectateur reproduit en fait le schéma de l'homme tout-puissant, dominant la Nature (qui se retrouve dans tout le discours du film). On peut l'interpréter comme un témoignage de l'hubris caractéristique de la société industrielle - or celle-ci se trouve à l'origine de la crise écologique.

Cette prise de distance entre l'homme-spectateur et le paysage-spectacle est d'ailleurs présente dès le générique du film, avec une vue de la Terre depuis l'espace. Cela rappelle les premières photographies de la Terre prises depuis la lune, notamment celle d'un « lever de terre » prise lors de la mission Apollo 8 (illustration 10). Ces photos sont souvent présentées comme la source d'une prise de conscience écologique : elles seraient la preuve visible de l'unité de destin de l'humanité sur notre planète, unique domaine du vivant dans l'infini de l'espace. Ces images représentent pourtant la prise de distance maximale que l'homme puisse prendre avec son environnement. Et elles valorisent la puissance technique extraordinaire qui a permis d'atteindre la Lune, suggérant la

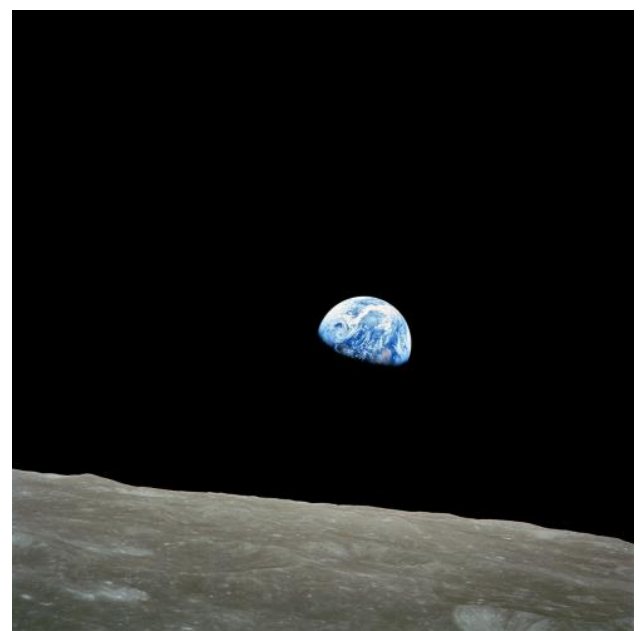

Illustration 10 : NASA, mission

Apollo 8, Earthrise (Lune, 1968)

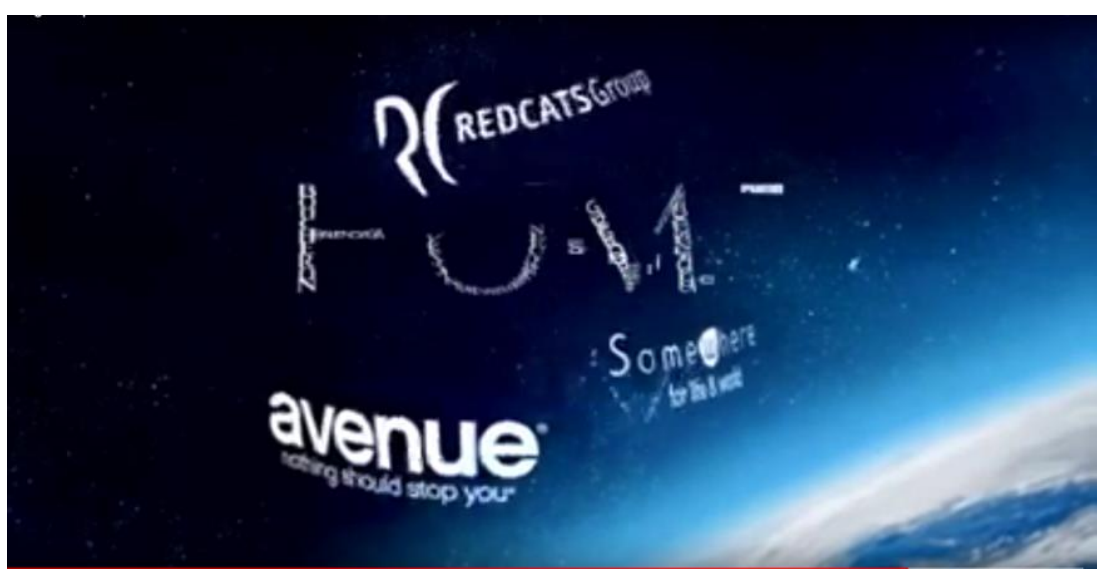

Illustration 11 : Yann Arthus-Bertrand, générique du film Home (France, 2009)

possibilité de s'extraire du milieu terrestre.

On remarquera d'ailleurs que l'utilisation répétitive de cette image de la Terre comme argument commercial sur tout type de produit a fini par en faire un cliché éculé. Extraite de son contexte, cette image perd son impact, et dévoile le détournement de ce modèle paysager dans le cadre de la société de consommation. Cette autre dimension du paysage-spectacle est mise en scène (bien que ce soit surement involontaire) dans le générique du film Home (illustration 11). Le titre du film est progressivement construit à partir de signes flottant autour de la planète : il s'agit en fait de logos, ceux des marques sponsors du film.

Le paysage-spectacle est d'ailleurs souvent utilisé dans un objectif publicitaire, à travers des images de paysages naturels, sauvages et grandioses. Cela permet d'associer des émotions agréables à un produit, un service, une entreprise ou même une institution. Mais surtout, l'évocation de la nature permet de créer un effet-écologie, utilisé comme argument de vente ou de persuasion (que les caractéristiques écologiques vantées soient réelles ou non).

En terme d'aménagement, on retrouve ce modèle paysager à l'œuvre dans les stratégies de protections, par exemple dans réserves naturelles et les parcs naturels nationaux. La dimension spectaculaire de ces paysages protégées est alors utilisée pour développer le tourisme. Mais paradoxalement, ces images et pratiques de protection entretiennent la représentation illusoire d'une nature sauvage, préservée, et contribuent donc à masquer l'impact réel des activités humaines sur la nature et les paysages. 
En résumé, le modèle du paysage-spectacle est constitué par la combinaison de l'esthétique pittoresque du paysage-décor avec l'esthétique sublime du paysage-émotion. En contradiction avec le message écologiste qu'il accompagne souvent, il perpétue une vision illusoire de la nature et une prise de distance entre l'homme et son environnement. Cet effet-écologie creux constitué par le paysage-spectacle est caractéristique de la dimension spectaculaire de la société de consommation, dans le sens que Guy Debord a théorisé avec le concept de société du spectacle.

\section{Le paysage - catastrophe}

On peut cependant remarquer que les représentations de la nature proposées dans ce type d'images spectaculaires ne sont pas toujours aussi paradisiaques. Ainsi, toute une partie du film Home est consacrée à des séquences montrant l'impact négatif des activités humaines sur la planète et sur les paysages : déforestation, urbanisation, pollution, etc. C'est en quelque sorte l'envers du décor qui est alors donné en spectacle, à travers des paysages de ruine et de destruction. L'objectif de diffusion d'une conscience écologique est alors envisagé différemment : si l'amour de la nature ne suffit pas à la préserver, la peur de la catastrophe écologique nous y contraindra peut-être ?

Christian Chelebourg a étudié la médiatisation de ce discours écologiste de la crise dans la littérature, et surtout dans le cinéma contemporain ${ }^{11}$. Il nomme écofiction ce modèle narratif et esthétique, qui particulièrement présent dans les films-catastrophe américains. Les paysages de l'écofiction me semblent constituer une forme particulière du paysage-spectacle. J'y repère l'émergence d'un nouveau modèle paysager, que j'appelle le paysage-catastrophe.

Dans une conférence sur le thème de la fiction paysagère, Christian Chelebourg expliquait que « le paysage écofictionnel se caractérise par une esthétique de la trace, qui expose l'empreinte de l'homme sur la nature pour mieux la dénoncer. Il renouvelle ainsi le paysage post-apocalyptique en le mettant au service de l' "heuristique de la peur". Je m'attacherai à mettre à jour les ressorts de la trouble fascination qu'exercent les représentations de ruines urbaines, les visions d'une planète

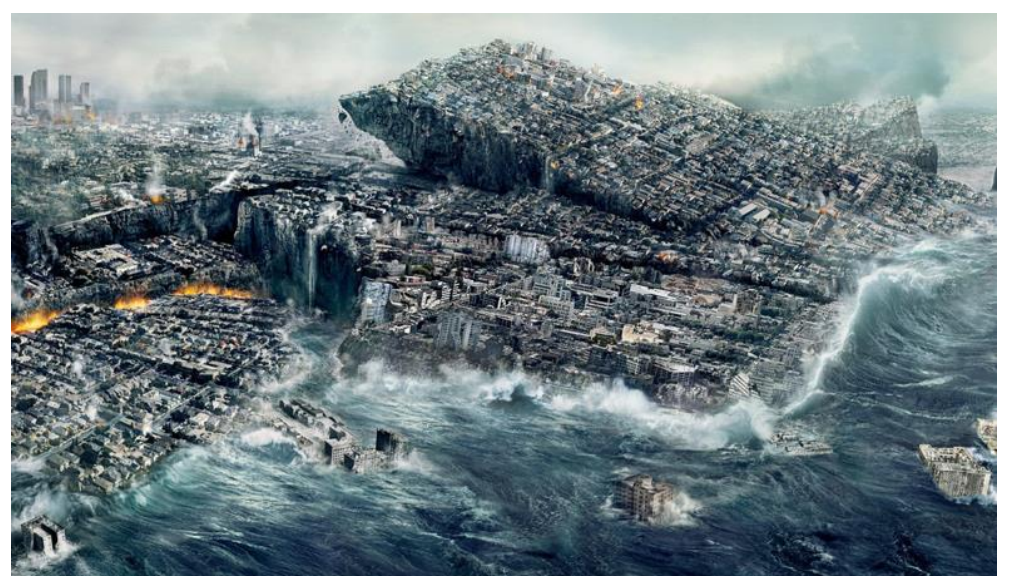

IIlustration 12 : 2012, de Roland Emerich (USA, 2009)

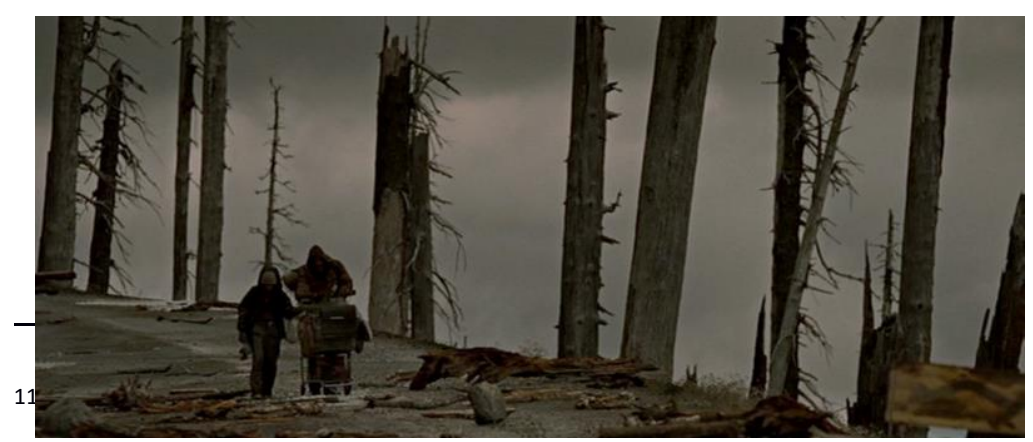

Illustration 14 : La Route, de John Hillcoat (USA, 2009)

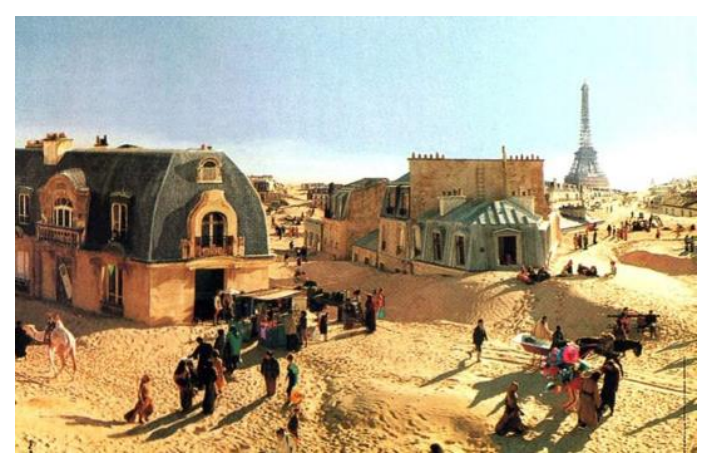

Illustration 13 : Peut-être, de Cédric Klapisch (France, 1999)

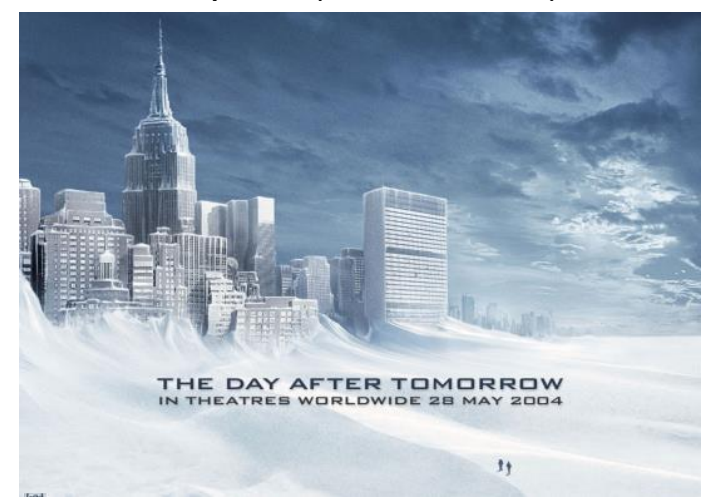

IIlustration 15 : Le jour d'après, de Roland Emmerich (USA, 2004 ) 
ravagée par la pollution, abrasée par la désertification ou rendue à la puissance des éléments. » ${ }^{12}$

Voici quatre exemples de ce modèle du paysage-catastrophe, dans quatre films. Dans 2012, on assiste à la destruction de Los Angeles par des séismes (illustration 12). Peut-être met en scène la ville de Paris, recouverte de sable suite au réchauffement climatique (illustration 13). La Route se déroule 10 ans après une explosion apocalyptique, dans un monde privé de végétation comme de civilisation (illustration 14). Dans Le jour d'après, un changement climatique extrêmement rapide et violent a enseveli New-York sous la neige et la glace (illustration 15).

Certains éléments paysagers sont récurrents dans les écofictions : ruines urbaines, pollution et catastrophes naturelles diverses (séisme, ouragan, glaciation, sécheresse, inondations). Bien que ce ne soit pas le cas dans tous les films-catastrophes, dans les écofictions c'est l'activité humaine qui est à l'origine de ces destructions. Cependant ce n'est pas le processus écologique qui est montré, mais uniquement son résultat, sous forme d'une esthétique de la ruine.

On retrouve dans ces représentations une esthétique du pittoresque, par exemple avec la valorisation de monuments célèbres. On retrouve également l'esthétique du sublime et tous ses paradoxes : le jeu avec les émotions, la prise de distance qui nous permet d'apprécier ces scènes avec une «exquise terreur », mais aussi l'utilisation principalement symbolique des éléments naturels. La nature est d'ailleurs représentée sous une forme particulièrement simplifiée : dans chacun de ces films, le paysage est dominé par la présence d'un élément, associé à une couleur majoritaire : l'eau bleue, la poussière grise, le sable jaune, la neige blanche... On remarquera aussi que les scènes se déroulent généralement en milieu urbain, et que ces éléments représentent chacun une catastrophe naturelle. La relation entre l'humain et son environnement qui transparait ici n'est en fait qu'un remake du combat de la Civilisation contre la Nature. La peur de la catastrophe écologique ne serait-elle donc qu'une résurgence de la peur de la nature ?

Le dénouement de ces films confirme cette hypothèse. En caricaturant à peine, deux fins sont possibles : soit l'homme est dépassé par la puissance de la nature, et la civilisation, voire l'humanité est détruite. Soit un héros parvient, grâce à des connaissances scientifiques et des prouesses technologiques à redevenir maître de la nature, et la civilisation - voire la planète entière, est sauvée... Cette exaltation de l'hubris humaine se traduit dans les représentations paysagères par le même point de vue extérieur et surplombant que dans les modèles du paysage-décor et du paysagespectacle, de même que dans la représentation symbolique, simpliste et caricaturale des milieux terrestres.

En résumé, le modèle du paysage-catastrophe expose les conséquences écologiques de l'activité humaine, à travers une esthétique de la ruine, à la fois pittoresque et sublime. Elle présente en fait une image caricaturale de la Nature, dans le scénario d'une opposition à la Civilisation. L'heuristique de la peur ici convoquée se détache de toutes considération écologique pour s'autonomiser en tant que représentation spectaculaire.

On peut donc s'interroger sur la pertinence de ce modèle en tant que vecteur d'un discours sur l'écologie. Des scientifiques ont relevé le caractère incohérent et peu crédible des scénarios de ces films-catastrophes. On pourrait en dire autant des représentations paysagères qui les illustrent. Leur intérêt semble plutôt résider dans leur impact émotionnel, qui en fait un spectacle particulièrement attrayant et distrayant - pour les spectateurs... et rentable pour ceux qui les diffusent.

\footnotetext{
${ }^{12}$ Chelebourg, Ch. « Paysages post-apocalyptiques et paysages écofictionnels ». Conférence au colloque

" Écofictions, fictions paysagères », Sorèze, février 2013.
} 


\section{PISTES VERS UNE ÉCOPOÉTIQUE PAYSAGÈRE}

Nous avons jusqu'ici traversé quatre modèles paysagers : le paysage-décor, le paysage-émotion, le paysage-spectacle et le paysage-catastrophe. Il semble que ces modèles couvrent la majorité des représentations paysagères fréquemment associées à un discours sur l'écologie. Mais nous avons remarqué qu'il traduisent en fait une fiction verte qui n'a pas de relation directe avec une vision du monde proprement écologique. La question se pose alors : comment aller au-delà de cette fiction verte? Quels nouveaux modèles paysagers peuvent être convoqués pour avancer vers une écopoétique?

Alors que mon étude de la fiction verte se réalisait dans des cadres habituels (pour une étude en art, en France), ces questions m'ont entrainée dans des mondes que je connais moins. Je me suis intéressée à d'autres disciplines, comme l'écologie scientifique, l'anthropologie ou la géographie, et à d'autres cultures, comme celles de l'antiquité chinoise, des indiens d'Amazonie ou des aborigènes d'Australie. Pour vous présenter mes hypothèses de recherche, je dois donc m'avancer dans des domaines que je ne maîtrise pas, mais qui m’ont inspiré de nouvelles idées.

\section{Le paysage - système}

La première piste que j'ai suivie, à la recherche d'une approche purement écologique du paysage, est de revenir à une approche scientifique de l'écologie. Car avant de se déployer dans des discours politiques, philosophiques et autres, l'écologie se définit comme la science qui étudie les relations entre les êtres vivants et leur milieu. Ce milieu est constitué d'éléments physiques (eau, air, terre...), d'autres êtres vivants (animaux, végétaux, bactéries...) et des conditions géographiques locales (climat, luminosité...). Le modèle paysager qui en émerge est évidemment propre à une vision du monde naturaliste et scientifique, toutefois différente de l'approche cartésienne associée au paysagedécor.

On entend parfois dire que l'écologie scientifique n'a rien à voir avec la question du paysage, car elle ne s'intéresserait qu'à l'environnement (ce qui désigne une réalité physique) et non à sa perception ou sa représentation en tant que telle. On y trouve pourtant des représentations paysagères à tous les niveaux.
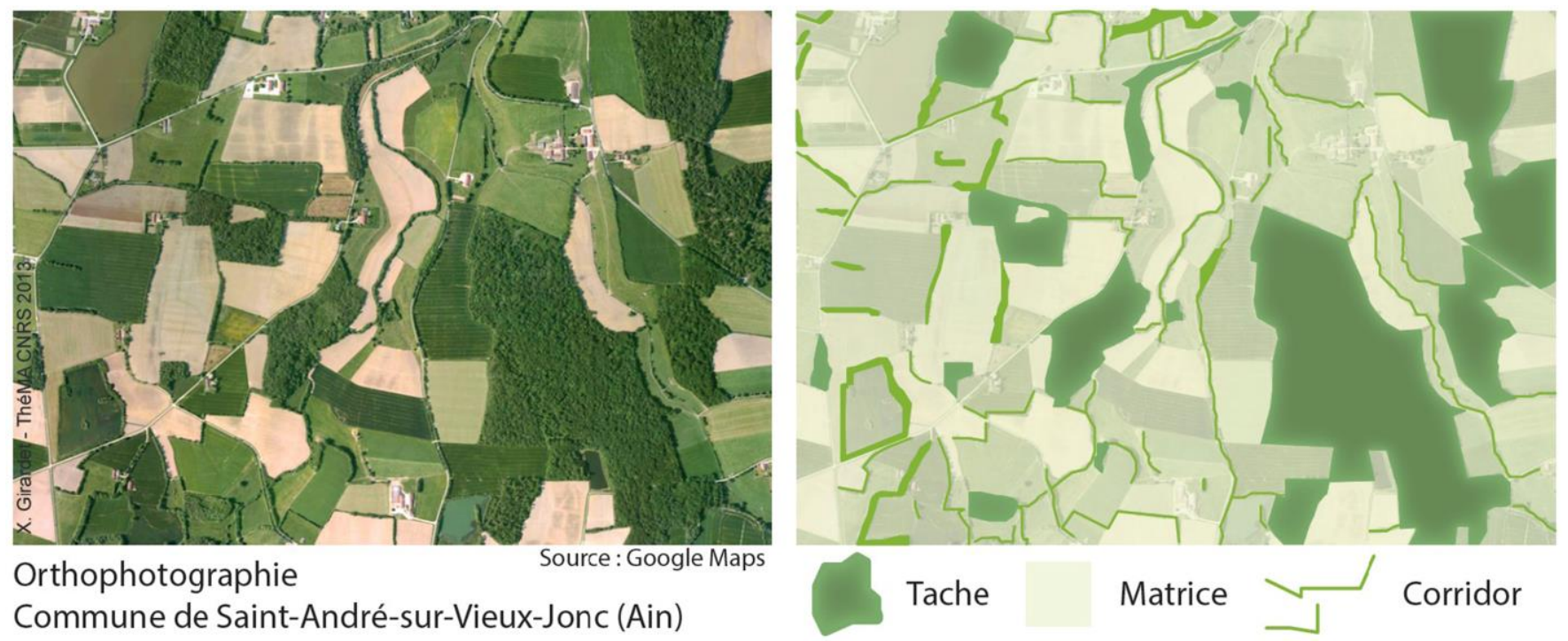

Illustration 16 : photographie et représentation schématique d'un écocomplexe paysager, Graph4ecology (France, 2017) 
Les écologues eux-même en utilisent pour effectuer leurs observations et les retranscrire, notamment sous forme de photographies (souvent aériennes) et de cartographies (illustration 16). On trouve aussi des dessins et croquis, illustratifs ou schématiques, dans la présentation des résultats de la recherche et dans les publications à visée pédagogique, livres pour étudiants ou de vulgarisation $^{13}$ (illustration 17).

Enfin, des représentations paysagères sont utilisées quand l'écologie est appliquée à l'aménagement du territoire (paysagisme, agriculture, plans d'urbanisme et schémas de cohérence territoriale, parcs naturels...), sous forme de photographies de constat, cartographies, croquis et plans de conception, par exemple pour la représentation des «trames vertes et bleues » (illustration 18).

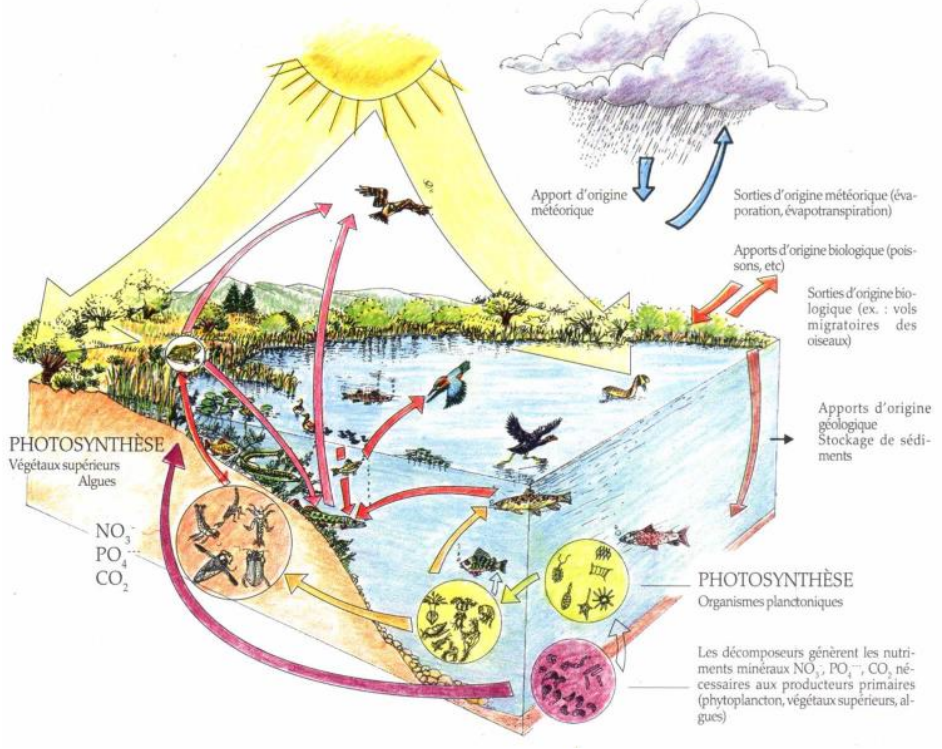

Illustration 17 : illustration schématique d'un écosystème lacustre, Bernard Fishesser et MarieFrance Dupuis-Tate, Le guide illustré de l'écologie (France, 2007)

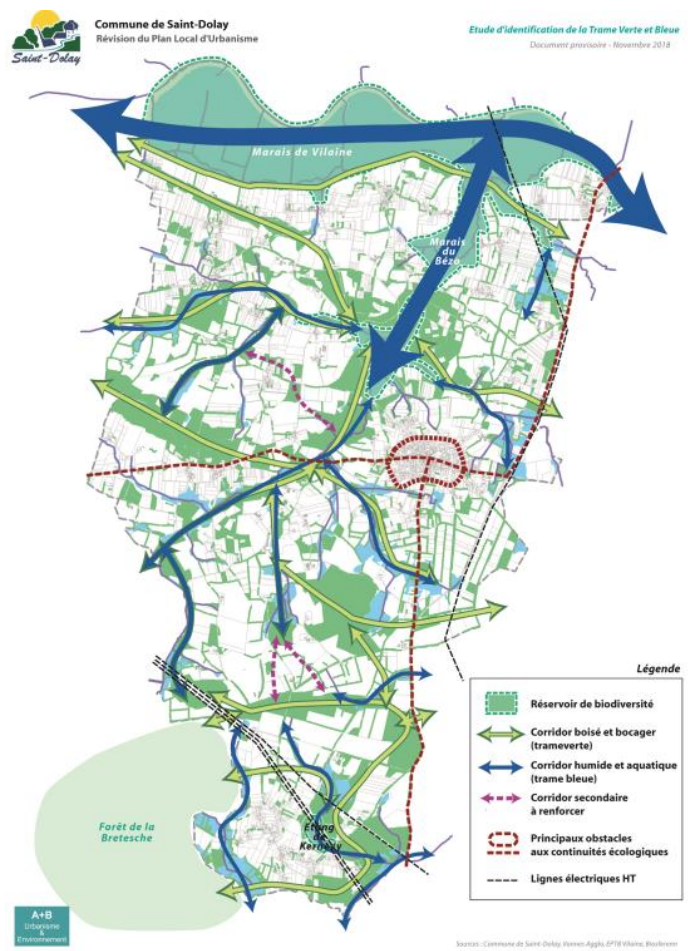

IIlustration 18 : Trame verte et bleue, PLU commune de Saint-Dolay (2018)

Les paysages sont représentés à différentes échelles, notamment dans trois branches de l'écologie. La synécologie étudie les écosystèmes. L'écosystème est un concept théorique représentant l'ensemble systémique formé par les interactions entre des êtres vivants et un environnement physique, formant une unité fonctionnelle. Elle se traduit d'un point de vue paysager par un milieu local identifié, comme une forêt, un lac, un estuaire... À l'échelle supérieure, l'écologie du paysage étudie les interactions entre différents écosystèmes. Le terme de paysage est alors utilisé (on dit aussi éco-complexe), pour désigner l'ensemble systémique formé par différents écosystèmes adjacents. Enfin, l'écologie globale étudie la biosphère, comme un écosystème à l'échelle planétaire. Si l'on ne trouve pas vraiment de représentations d'ensemble de la biosphère (que l'on pourrait qualifier de méga-paysage), on trouve le concept de biome, qui représente un ensemble écosystémique identifiable à l'échelle planétaire. Les différents biomes sont définis comme des catégories paysagères telles que le désert, l’océan, la savane ou la toundra.

\footnotetext{
${ }^{13}$ Fischesser, B. et Dupuis-Tate, M.-F. Le guide illustré de l'écologie.
} 
Une caractéristique marquante de ces représentations paysagère est l'association d'une représentation spatiale (photo, carte ou dessin) et de schémas représentant des interactions systémiques (flèches), comme on en trouve souvent dans les cartes géographiques. Ceci est révélateur de l'affinité très forte entre la science écologique et la pensée systémique. Le paysage perçu est généralement considéré par l'écologue comme un système. Par exemple, dans un paysage de forêt, il verra un écosystème forestier, dans une vue aérienne, un écocomplexe paysager. C'est pourquoi je propose de nommer ce modèle le paysage-système.

On remarquera que les représentations d'écosystèmes ou de biomes enrichissent les représentations paysagères classiques grâce à des éléments visuels (schémas systémiques et cartographie), que l'on peut qualifier de graphisme d'information. Il n'apparait pas là une esthétique nouvelle, mais plutôt la reprise des codes graphiques de la géographie. L'écologie du paysage est particulièrement intéressante en ce qu'elle invente de nouveaux concepts de lecture du paysage. La structure spatiale du paysage est ainsi définie en termes de matrice, tache, corridor et lisière. Les paramètres fonctionnels sont aussi identifiés pour caractériser un écocomplexe paysager : on observe et on mesure sa diversité, son hétérogénéité, sa complexité et sa connectivité. Ces notions entrainent de nouveaux schèmes de perception et de représentation du paysage.

Ce modèle du paysage - système renouvelle donc le regard que nous portons sur les paysages qui nous entourent et participe ainsi à une évolution de notre vision du monde. Il s'avère aussi opérationnel pour les projets d'aménagements paysagers (par exemple, dans la multiplication des projets de trames vertes et bleues) et contribue donc aussi à une évolution des pratiques.

Cependant, l'origine scientifique de ce modèle marque ses limites sur le plan esthétique. C'est d'ailleurs pourquoi les écologues font appel à des artistes pour illustrer les ouvrages de vulgarisation, dans l'intention de combler l'écart entre la représentation scientifique du système et la dimension esthétique et sensible du paysage. Cette solution semble cependant insuffisante pour traduire la relation au monde particulière induite par une perception du monde écologique. On constate ainsi une omniprésence du point de vue extérieur et dominant que nous avons identifié dans les modèles de la fiction verte paysagère, typique d'une vision du monde naturaliste.

\section{Du paysage-système au paysage-cosmos : Gaïa}

La science naturaliste a aussi parfois donné lieu à d'autres représentations originales, par exemple avec l'Hypothèse Gaïa de James Lovelock ${ }^{14}$. Ce physicien défendit l'idée consistant à voir la Terre comme un écosystème planétaire, plus exactement un système auto-régulé formé par les interactions entre la biosphère et l'environnement physique : géosphère, atmosphère et hydrosphère (illustration 19). Il présenta cette idée en disant que «la Terre est un organisme vivant », et utilisa pour la désigner le nom du Gaïa. Gaïa était une divinité primordiale dans la mythologie grecque, représentant la Terre-Mère (illustration 20), et mariée à Ouranos, dieu du ciel. L'idée d'une planète vivante était une métaphore utilisée par Lovelock pour évoquer l'unité systémique entre les êtres vivants et leur environnement physico-chimique à l'échelle planétaire, considérant l'autoorganisation comme une propriété émergente de ce système. Mais elle a souvent été prise au premier degré. Ce qui lui a valu les critiques de scientifiques, mais a aussi donné naissance à un mouvement spirituel new-age écoféministe, considérant Gaïa comme une entité douée de conscience. On voit alors apparaître dans la culture populaire occidentale de nouvelles représentations de la «Terremère », images hybrides issues du croisement entre la science contemporaine la plus en pointe et la résurgence de mythes cosmologiques anciens (illustration 21). 


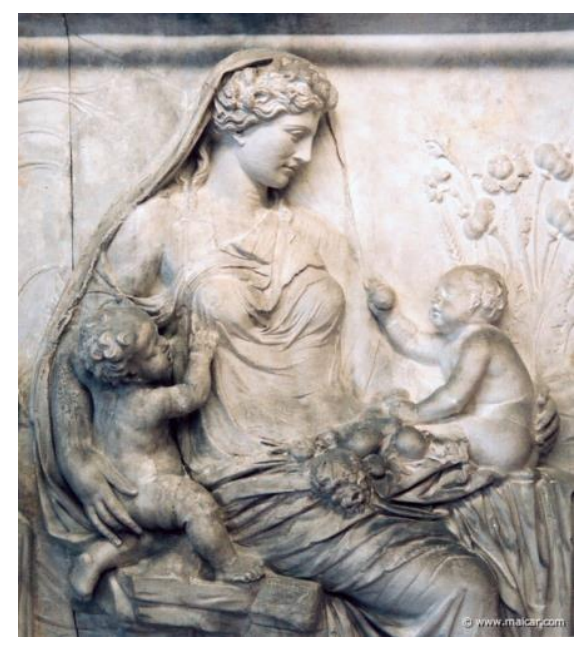

Illustration 20 : Bas-relief romain représentant Tellus, équivalent de Gaïa, déesse grecque de la Terre

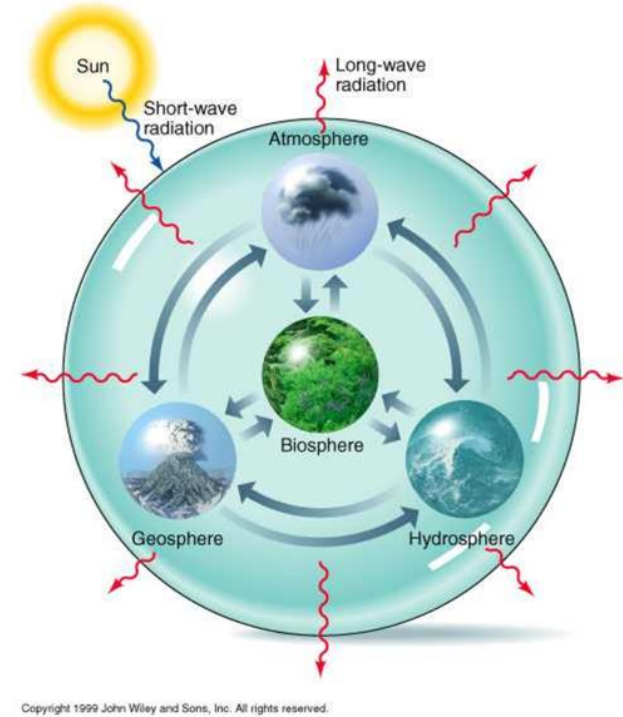

Illustration 19 : L'hypothèse Gaïa de James Lovelock : la terre comme système auto-régulé

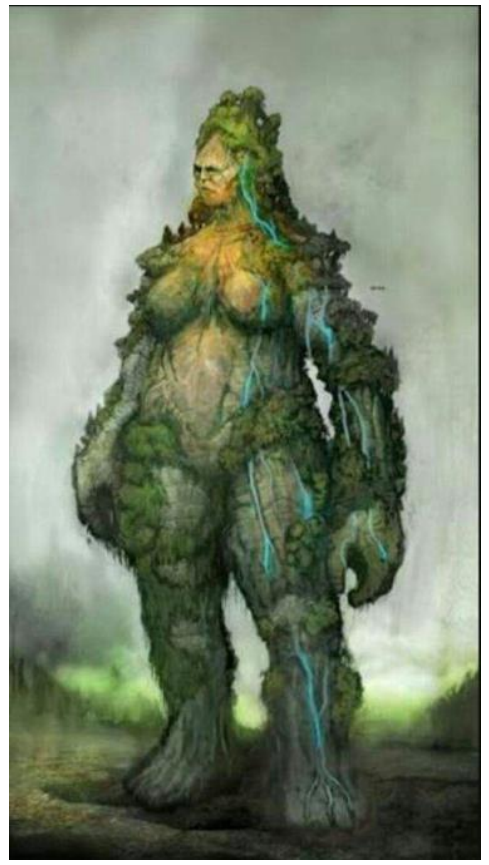

Illustration 21 : Image de Gaïa comme déesse de la terre dans un jeu vidéo

\section{Le paysage - cosmos}

Reprenons donc notre recherche d'autres modèles paysagers, vers une piste qui soit non seulement écologique mais également poétique. Et s'il fallait, pour trouver des représentations différentes, déplacer notre regard à l'extérieur de la société occidentale ? Cela suppose d'adopter une approche du paysage élargie, afin d'inclure des pratiques et des représentations que l'on observe dans d'autres sociétés que celles identifiées comme strictement paysagères. L'anthropologue Philippe Descola a approfondi cette proposition dans son cours donné au Collège de France sur «Les formes du paysage ${ }^{15}$.

Je me suis inspirée pour identifier ces nouveaux modèles paysagers de la grille de lecture des différentes visions du monde qu'il a proposé, en identifiant quatre ontologies ${ }^{16}$. Le naturalisme désigne l'approche occidentale moderne, fondée sur la séparation entre Nature et Culture. L'ontologie analogiste se représente le monde comme un ensemble organisé par des relations analogiques. Elle est propre aux grandes civilisations agricoles, ayant une structure sociale hiérarchisée et une religion polythéiste. On la trouve en Chine, en Amérique (Maya, Aztèque, Inca), en Afrique de l'Ouest, et autour de la Méditerranée dans l'Antiquité (Grecs, Romains, Egyptiens...). Ce qu'il nomme l'ontologie totémiste se réfère uniquement aux peuples aborigènes d'Australie. Je reparlerai de la vision du monde animiste.

Le terme d'ontologie me semble bien décrire les visions du monde naturalistes et animistes, qui sont fondées sur le principe de l'être. Mais en ce qui concerne l'analogisme et le totémisme, j'utiliserai plutôt le terme de cosmologie, car leurs visions du monde sont plutôt fondées sur la notion de cosmos. Le cosmos est une représentation du monde en tant que totalité organisée, constituée par les inter-relations entre ses différents éléments. Un modèle cosmique représente par

\footnotetext{
${ }^{15}$ Descola, P. « Les formes du paysage ».

${ }^{16}$ Descola, P. Par-delà nature et culture.
} 
exemple les relations qui s'établissent entre des lieux, des humains et des êtres spirituels. En tant qu'unité fonctionnelle constituée d'inter-relations organisées, le principe même du cosmos pourrait être ré-interprété comme un modèle de pensée systémique. Mais à la différence du système scientifique, le modèle du cosmos donne du sens à ce qui est perçu, non seulement au niveau intellectuel, mais aussi dans notre appréhension sensible du monde.

J'ai trouvé dans ces cosmologies étrangères différents exemples de représentations paysagères, que je réunis pour constituer ce que je nomme le paysage-cosmos. Notons que je ne suis pas la première à emprunter cette piste. Augustin Berque la défriche consciencieusement depuis plusieurs dizaines d'années, et a montré l'intérêt de l'approche cosmique du paysage asiatique pour une pensée mésologique. ${ }^{17}$

Chez les peuples aborigènes d'Australie, le monde est organisé en différentes classes totémiques, qui regroupent les personnes, animaux, plantes et lieux en relation avec l'être du Rêve qui leur a donné naissance (ou dont ils sont l'incarnation). Le territoire est perçu comme trace de l'activité des Êtres du Rêve (par empreinte ou métamorphose). Cette transformation du territoire est ensuite représentée dans des schèmes visuels et des productions visant à reproduire de façon mimétique ces traces laissées dans le monde par les êtres du Rêve. Prenons par exemple l'œuvre de l'artiste aborigène Esau Lewis, intitulée Le rêve du kangourou (illustration 22). Cette peinture qui peut sembler presque abstraite montre en fait, dans une même image : l'Être du Rêve (ici le kangourou) en train de parcourir (et donc créer) le territoire ; les sites nés de cette action (trous d'eau, collines, pistes); et des emblèmes du groupe totémique associé à cet être et à ces sites. C'est la représentation topographique d'une narration, qui fait écho au trajet parcouru par les membres d'un clan pour apprendre leur histoire. Cette peinture est donc à la fois une carte, un récit cosmologique et une sorte d'armoirie de clan. Il s'agit bien de paysage, en tant que représentation d'un lieu dotée d'une signification, et ce paysage présente l'organisation du cosmos.

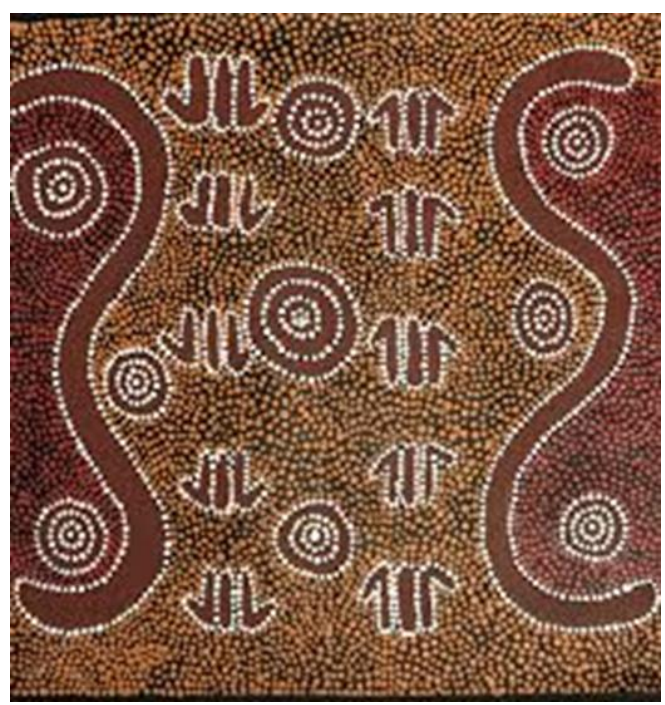

IIlustration 22 : Esau Lewis, Kangaroo Dreaming (Centre Australie, fin 20 e siècle)

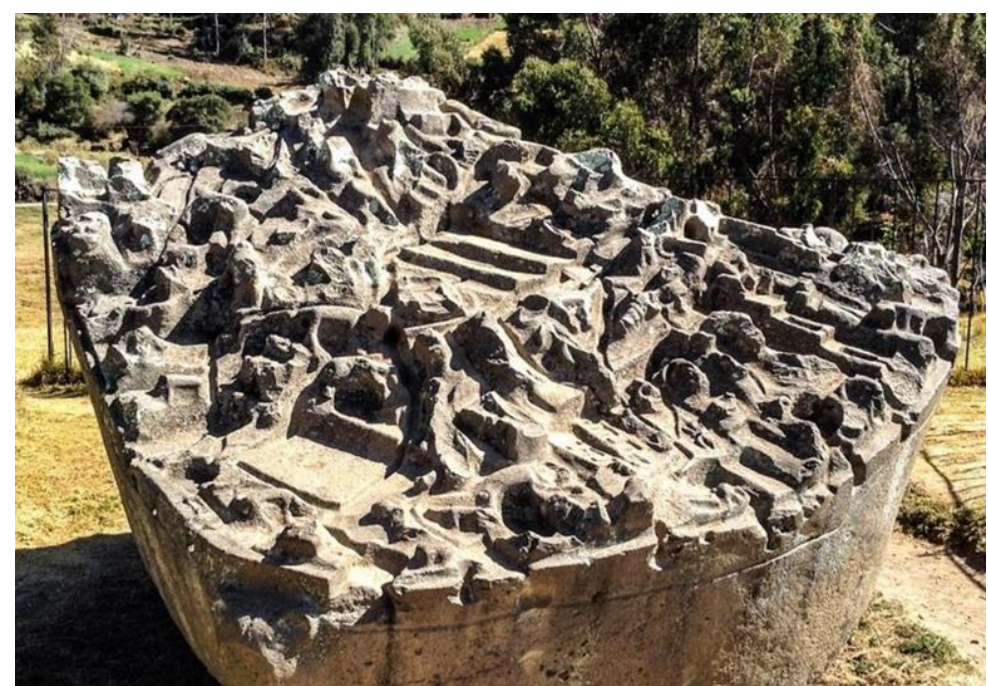

Illustration 23 : Pierre de Sayhuite (temple Inca, Pérou, entre $13 e$ et 16 e siècle)

Dans la civilisation Inca, le schème cosmique est fondé sur le modèle de la circulation de l'eau dans les montagnes. Les représentations des rivières et des montagnes sont nombreuses, sous forme

${ }^{17}$ Berque, A. Poétique de la terre. 
d'aménagement de site ou de reproduction d'un territoire en miniature. La plus célèbre est la Pierre de Sayhuite (illustration 23), trouvée dans un temple au sommet d'une pyramide, lieu d'un culte lié à l'eau. On peut l'interpréter comme un schéma topographique, une maquette d'ingénierie hydraulique, ou encore une représentation mythologique intégrant des figures animales et des divinités. Mais il est probable qu'elle représente tout cela en même temps, puisque dans la cosmologie Inca ce sont les mêmes principes qui s'expriment dans la morphologie des montagnes et des cours d'eau, l'aménagement du territoire, les relations sociales et avec les dieux. Ce type de production peut être considéré comme paysage, non en tant que représentation iconique, ni même symbolique, mais en tant qu'indice témoignant de l'organisation cosmique du monde.

De l'autre côté de la planète, l'expression "montagnes et eaux » (shanshui en chinois) désigne une forme de représentation paysagère, propre à la peinture taoïste, dont on peut voir un exemple dans une peinture de Wang Quan (illustration 24). Cette image ressemble plus aux représentations paysagères qui nous sont familières. Mais il ne faudrait pas croire qu'il s'agisse, comme en occident, d'une représentation mimétique d'un paysage naturel, tel qu'il est perçu par un sujet qui le contemple. L'art du shanshui, d'inspiration taoïste, consiste en fait à "capter le souffle de l'univers $»^{18}$. Le peintre exprime, à travers la peinture de scènes paysagères imaginées, les relations dynamiques qui organisent le cosmos, et se déploient de la même manière dans son esprit que dans le monde visible. Pour simplifier, on peut dire que les montagnes représentent le principe yang (actif), les eaux le principe Yin (réceptif), et la brume le Chi (l'énergie, la dynamique qui anime les relations entre yin et yang). L'humain est aussi toujours présent dans ces représentations, sous forme de personnages ou d'habitations. On remarquera que contrairement aux modèles paysagers de la modernité, l'humain n'est pas placé au premier plan ou au-dessus du paysage, mais y est intégré en tant qu'un élément parmi d'autres de cet équilibre cosmique.

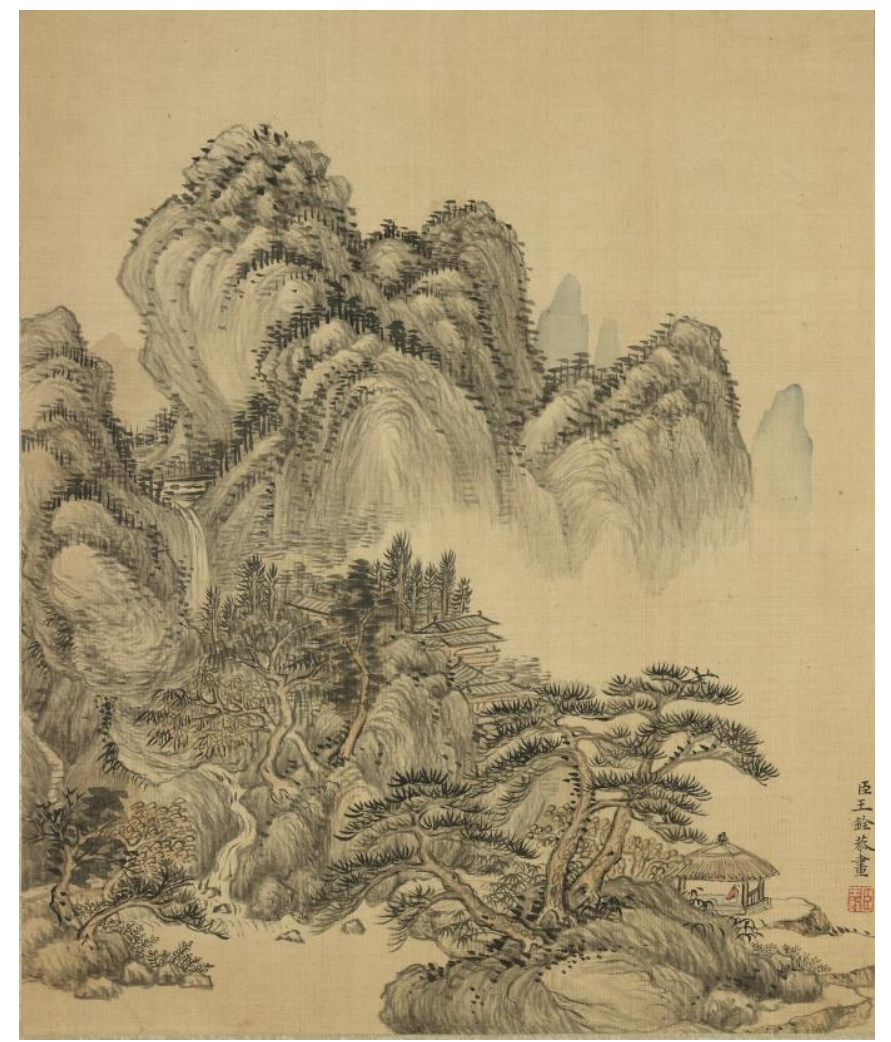

Illustration 24 : Peinture de sanshui de Wang Quan (Chine, 12e / 13e siècle)

\footnotetext{
${ }^{18}$ Voir à ce sujet : Escande, Y. Montagnes et eaux.
} 
En résumé, l'ouverture à des formes de représentations paysagères hors de la modernité occidentale nous permet de découvrir une autre manière de voir le monde. La distinction entre Nature et Culture fait place à une vision du monde comme cosmos, ou totalité organisée. On peut tirer des représentations paysagères nées de ces cosmologies totémiste ou analogiste une inspiration pour constituer un autre modèle paysager : le paysage-cosmos. Ce modèle, en abolissant la frontière entre nature et culture, et en replaçant l'humain au sein d'une organisation systémique qui le dépasse, nous permet d'imaginer des représentations paysagères en accord avec les théories de la science écologique, tout en leur conférant une signification et une dimension poétique ou spirituelle.

\section{Du paysage - cosmos au paysage - vivant : Artificial wonderland}

L'œuvre de Yang Yongliang, photographe et plasticien chinois, présente un cas intéressant de réinterprétation de ce modèle du paysage-cosmos dans l'art contemporain, guidé par une pensée de l'écologie (illustration 25).

Cette œuvre reprend tous les codes visuels du shanshui traditionnel, mais les montagnes sont remplacées par des immeubles, les eaux sont polluées et les constructions humaines envahissent l'ensemble. Chaque photographie de cette série expose une problématique écologique, ici l'urbanisation démesurée. Le texte calligraphié dit «Laissons les collines être collines et les rivières être rivières », confirmant le message écologiste sous-jacent. Cette image provoque un sentiment mêlé de fascination et d'horreur. Il me semble que le malaise vient de la tension entre le modèle du paysage-cosmos (à travers la référence au shanshui), et le modèle du paysage-catastrophe (par la représentation photographique de l'envahissement urbain). Mais ce qui me semble encore plus marquant dans cette image, c'est l'absence totale de vie: on ne voit dans cette représentation paysagère ni végétation, ni animaux ni humain.

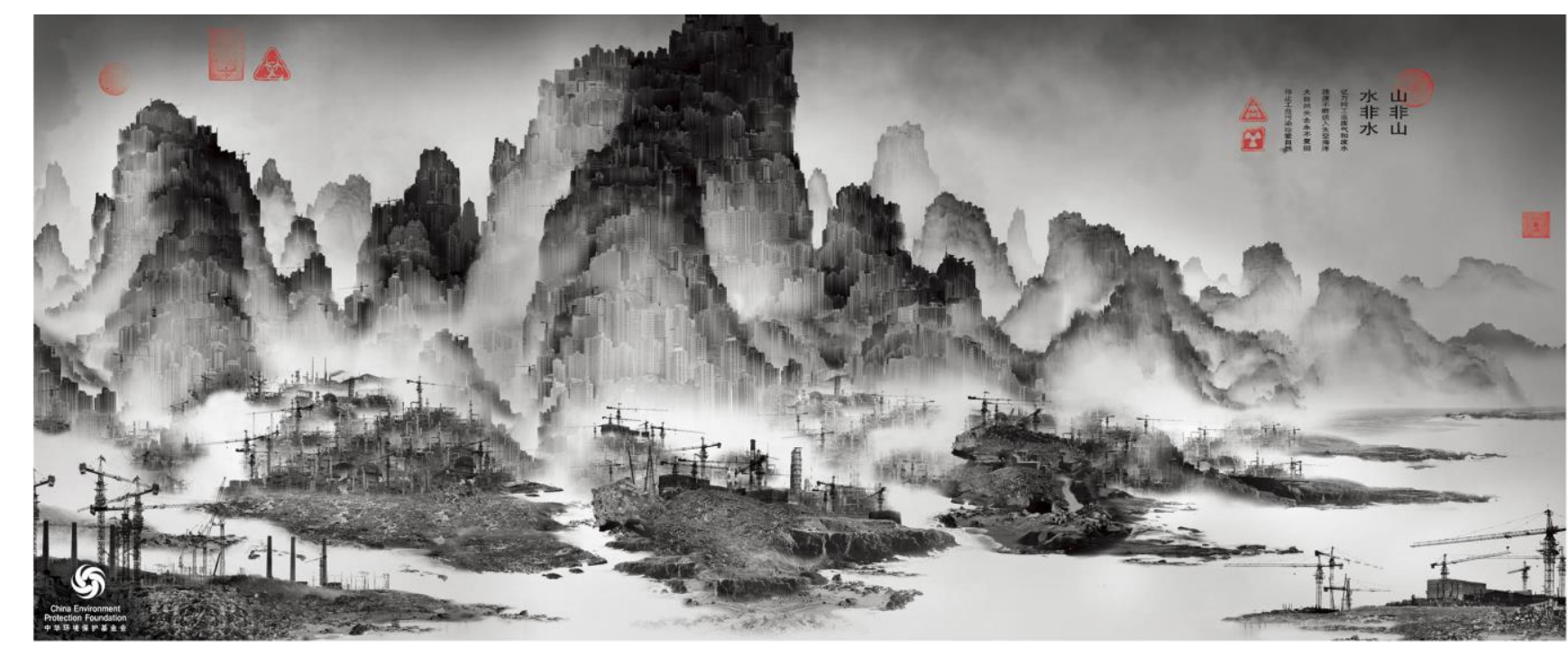

Illustration 25 : photomontage de Yang Yongliang, Pollution industrielle, de la série Artificial wonderland (Chine, 2013)

\section{Le paysage - vivant}

Si nous repensons aux modèles paysagers de l'écologie scientifique, nous constaterons que l'approche systémique est en fait révélatrice d'un questionnement sur les relations entre les êtres vivants et leur environnement. Pour aller plus loin dans ce qu'implique cet intérêt pour le vivant, je propose de chercher dans une autre direction. Le modèle ontologique que Philippe Descola désigne 
par le terme d'animisme ${ }^{19}$ est justement fondé sur le vivant. Cette approche se retrouve dans diverses sociétés, que l'on qualifie généralement de chasseurs-cueilleurs, avec des pratiques spirituelles de type chamanistes : par exemple en Amazonie, en Océanie, au nord de l'Amérique ou en Sibérie. Cette ontologie animiste est fondée sur une vision du monde comme réseau d'interrelations entre diverses sortes d'êtres : humains, animaux, végétaux et esprits.

L'ontologie naturaliste (la nôtre), est fondée sur une séparation nette entre les êtres vivants et les choses non-vivantes d'une part, et entre les êtres humains et non-humains d'autre part, d'où un modèle de représentation dans lequel on distingue les personnages des paysages. Ces distinctions n'existant pas de la même manière dans l'ontologie animiste, la perception de ce que nous nommons paysage est donc très différente.

On ne trouve pas dans les arts traditionnels de ces peuples d'équivalent exact de nos représentions paysagères, en-dehors des jardins (cas particulier sur lequel nous reviendrons). Par contre, on peut observer les créations de certains artistes contemporains, chez qui un héritage culturel de type animiste se mêle à une influence de la culture occidentale moderne. J'avance donc l'hypothèse que l'on peut percevoir dans ces œuvres une vision du monde animiste, traduite sous la forme d'une représentation paysagère, que je nomme le paysage-vivant.

Parmi les habitants indigènes de la forêt de Manu, au Pérou, certains artistes ou artisans développent depuis quelques années une forme originale de représentation paysagère sur des tentures murales en patchwork. La pièce intitulée Amazonian paradise (illustration 26) est une représentation de la forêt de Manu. Avant de qualifier cette scène de paysage, remarquons qu'elle est formé uniquement par des plantes et animaux : perroquets, toucan, paresseux, léopard, tapir, serpent, et une vingtaine de plantes différentes. Là où un regard européen chercherait des repères géographiques (terre, montagne, rivière...), cet artiste semble voir la forêt comme un réseau de vies entremêlées.

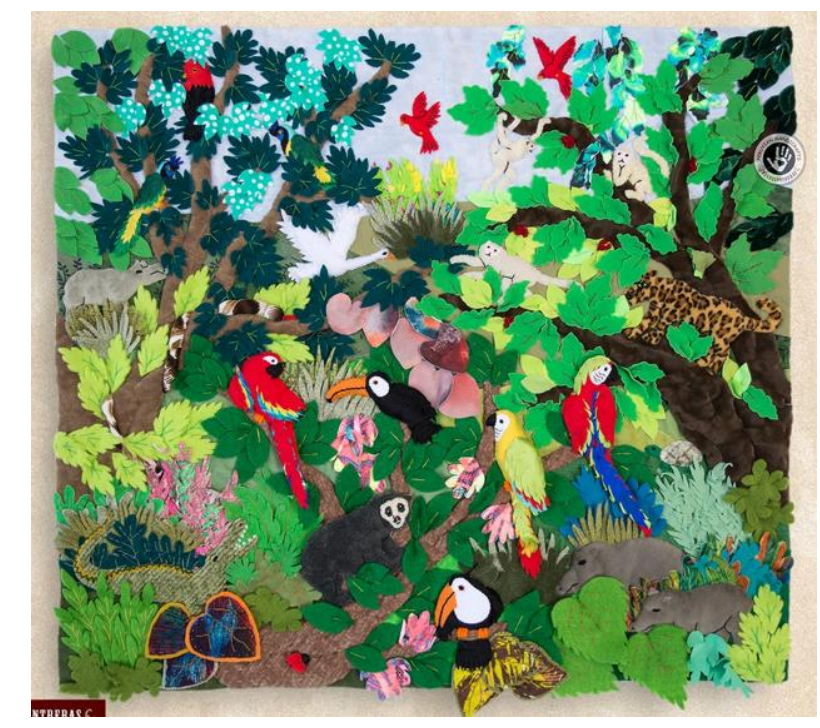

Illustration 26 : Amazonian paradise, tenture murale (patwork textile), par Edi Narrea (forêt de Manu, Pérou)

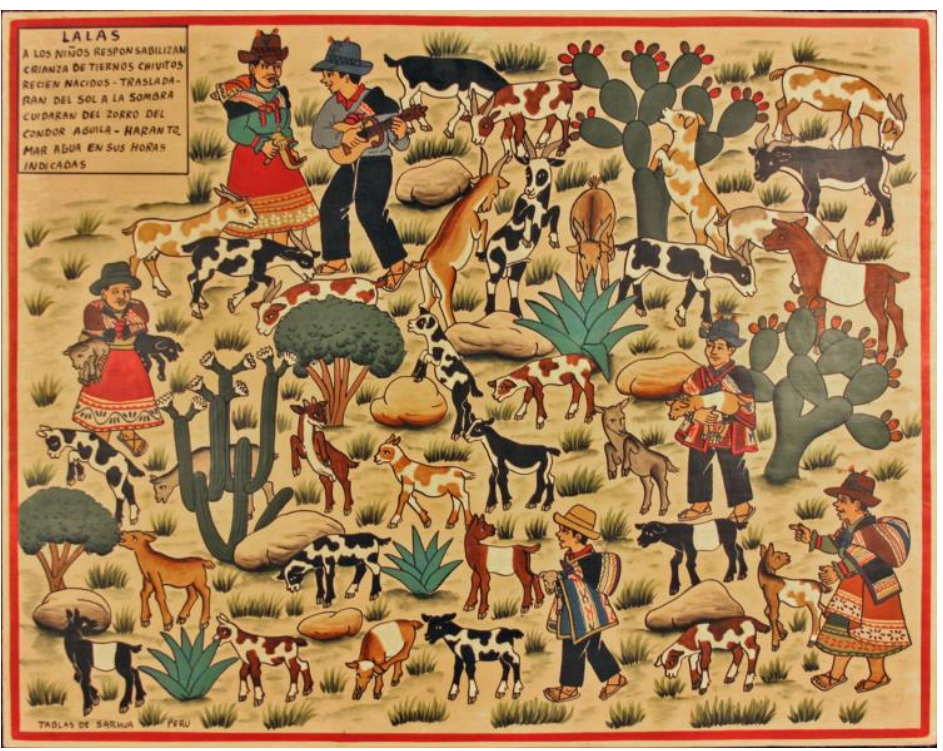

Illustration 27 : Table peinte de Sarhua (Pérou)

\footnotetext{
${ }^{19}$ Descola, P. Par-delà nature et culture.
} 
Peut-être issu d'une tradition Inca, les tables de Sarhua (illustration 27) sont peintes à l'occasion de la construction d'une maison pour un jeune couple. À l'origine, ces images représentant un arbre généalogique étaient peintes sur les poutres du toit. Aujourd'hui ce sont des tables, sur lesquelles les membres de la communauté sont représentés dans leur travail quotidien. On voit ici les enfants qui s'occupent des chèvres. La légende dit: «Les enfants sont responsables de l'élevage des petites chèvres nées récemment. Ils les déplacent du soleil à l'ombre et les protègent du renard, du condor et de l'aigle. Ils les font boire au bon moment. » Cela révèle des relations particulières entre humains, animaux et végétaux propre à toutes les communautés d'éleveurs. Cette image peut aussi être vue comme un paysage, puisqu'elle montre les éléments d'un environnement dans l'espace. Mais encore une fois, là où un peintre européen aurait probablement montré la terre, le ciel, l'horizon pour situer les personnages dans un espace, la scène n'est ici formée que par les relations entre divers êtres vivants : les enfants, les chèvres, mais aussi l'herbe qu'elles mangent, des arbustes dont elles mangent les feuilles, des cactus, aloès et figuiers de barbarie. C'est l'ensemble de ces relations entre humains, animaux et plantes qui constitue cette représentation paysagère.

Le peintre péruvien Roldán Pinedo crée des tableaux de type occidental, en utilisant et en représentant les savoirs traditionnels de la communauté Shipibo-Conibo, qu'il a hérité de ses parents et grand-parents. Il réalise ses peintures avec des pigments naturels tirés de la forêt, et l'abondance de détails dans la représentation des plantes et animaux démontre la connaissance intime qu'en a l'artiste. Sur le tableau intitulé Chullachaqui (illustration 28), les visages sur les troncs des arbres sont représentatifs de la manière dont son peuple se représente les arbres. Lorsque nous disons que ce sont des êtres vivants, nous comprenons généralement qu'il s'agit d'objets particuliers, car dotés d'une forme de vie (inférieure à la notre). Mais pour eux, cela signifie que ce sont des personnes, dont l'apparence cache un esprit similaire au notre - et avec qui nous pouvons donc communiquer. Le peintre entre en relation avec certaines plantes, qui lui disent elle-mêmes ce qu'il doit peindre et comment. Ses œuvres représentent des plantes et animaux sacrées de la forêt amazonienne, mais aussi les esprits et l'énergie de la forêt que lui montrent ces plantes. Lorsque je dis que cela représente un paysage-vivant, cela n'est pas seulement une manière rapide de désigner une scène associant divers êtres vivants. Cela signifie que le paysage lui-même est vivant.

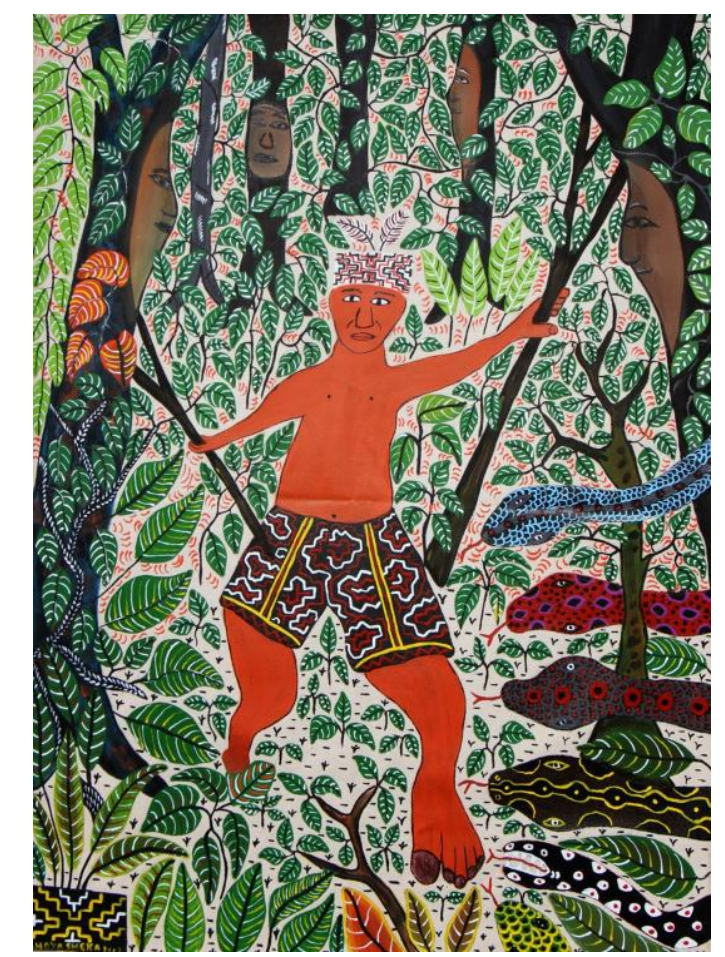

Illustration 28 : Chullachaqui, peinture de Roldán Pinedo (Pérou, 2014) 
Il peut nous sembler difficile d'appréhender cette approche face à ces représentations picturales, qui entrent en concurrence dans notre esprit avec les modèles paysagers habituels. Revenons-en donc à la manière donc s'exprime traditionnellement cette approche paysagère chez les peuples amazoniens, en amont de toute représentation : le jardin.

\section{Du paysage - vivant au paysage - milieu de vie : la forêt-jardin amazonienne et le jardin- forêt permaculturel}

Philippe Descola ${ }^{20}$ a étudié la question du paysage chez les Achuars, un peuple Jivaro vivant en Amazonie. Il remarque que si l'on ne trouve pas chez eux d'artefact que l'on puisse qualifier de représentation paysagère, ils cultivent des jardins. Et si l'on accepte de considérer la création de jardin vivrier comme une transfiguration paysagère (et non seulement les jardins d'ornement), cela nous ouvre une porte pour mieux comprendre ce modèle du paysage-vivant.

Le jardin occidental est caractérisé par sa clôture, qui établit une limite entre l'espace domestique $\mathrm{du}$ jardin et l'espace sauvage au-dehors (autrement dit entre la Culture et la Nature). Le jardin achuar (illustration 29) se déploie autour d'une habitation, sans frontière définie. Cela est lié à leur manière d'habiter et de cultiver la forêt. Une zone est défrichée et brûlée, puis cultivée et habitée pendant trois ans. Pendant ce temps, les habitants exploitent aussi la forêt dans un rayon d'une dizaine kilomètres, par leurs activités de cueillette et de chasse. Ensuite le processus est répété ailleurs. Il en résulte deux choses : le jardin proche de l'habitation est éphémère et cultivé en polyculture; la forêt elle-même est constituée d'espèces plantées auparavant dans les jardins redevenus forêt, ainsi que d'espèces favorisées par la chasse et la cueillette. On peut ainsi dire que le jardin n'est qu'un moment éphémère dans la vie de la forêt, ou que la forêt entière est un immense jardin... c'est pourquoi on a nommé ce modèle d'aménagement du territoire la forêt-jardin.

L'intérêt écologique de ce modèle est qu'il reproduit un fonctionnement écosystémique, marqué par une biodiversité importante, tout en fournissant aux habitants une grande variété de végétaux comestibles. Il n'y a ici aucune opposition entre Nature et Culture, nul besoin de protéger les cultures des nuisibles, ni de protéger les espèces sauvages de l'activité humaine, puisque celle-ci intègre et s'insère dans les équilibres écologiques de la forêt. C'est du moins une manière d'interpréter ces pratiques, dans l'optique d'une approche occidentale de l'écologie. Bien évidemment, les Achuars expliquent ces processus autrement: selon eux il y a bien une intentionnalité dans la gestion des plantes et animaux de la forêt, mais elle est due aux esprits dont elle est le jardin.

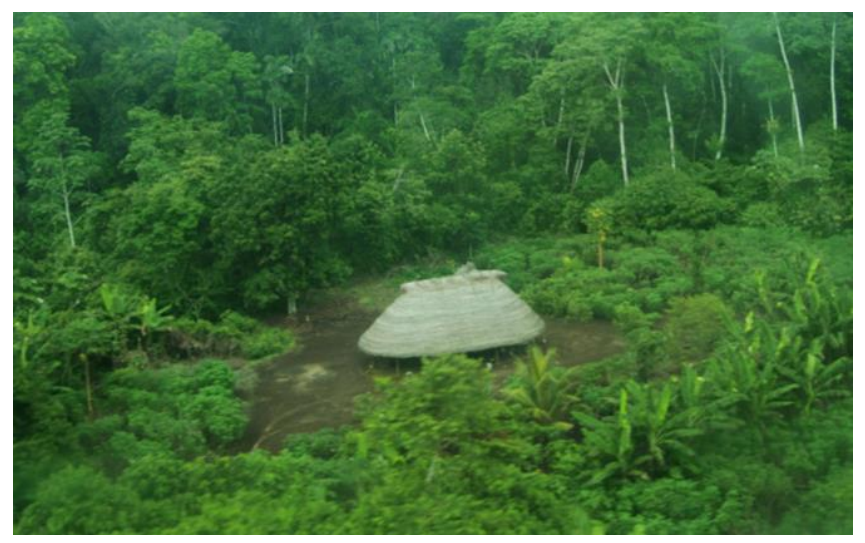

Illustration 29 : La forêt-jardin : une habitation achuar dans la forêt amazonienne

${ }^{20}$ Descola, P. « Les formes du paysage ».

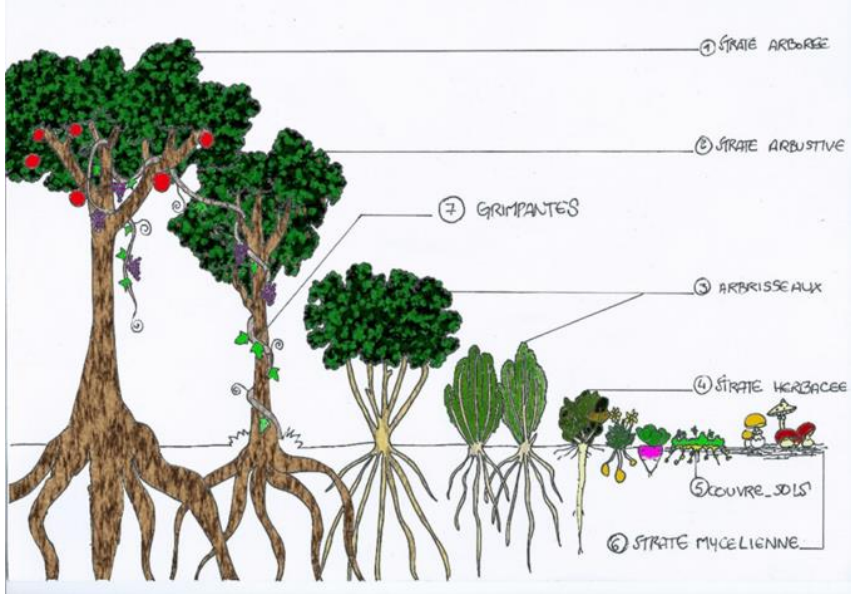

Illustration 30 : Le jardin-forêt en permaculture : schéma de principe 
En Angleterre, des spécialistes de l'agroforesterie et de la permaculture se sont inspirés de ces pratiques pour concevoir un nouveau modèle agricole nommé... le jardin-forêt. L'application de ce même principe de gestion est alors rattaché à des savoirs scientifiques, biologiques et écologiques, tout autant qu'à des savoir-faire de jardiniers et paysans. Le principe de la forêt-jardin consiste à associer différents végétaux comestibles de différentes tailles, de manière à reproduire l'organisation d'une forêt. Pour simplifier, on distingue sept strates de végétation : sous-terraine, couvre-sol, herbacée, arbrisseaux, arbustes, arbres et plantes grimpantes. Cette méthode permet de produire une grande diversité de légumes, fruits, noix, champignons, tout en profitant des associations entre les plantes, et sans besoin d'apporter ni eau, ni engrais, ni pesticide.

\section{Le paysage - milieu de vie}

\section{- Le jardin écologique}

Cette approche du jardin en continuité avec les processus écologiques est aujourd'hui de plus en plus mise en œuvre par des jardiniers et paysagistes, en général portés par une connaissance de l'écologie et un souci pour le vivant.

Jaap de Vries a ainsi conçu le jardin de Jacobstuin (illustration 31) par rapport à son évolution au cours des saisons, sans coupe rase à l'automne. II intègre une grande diversité végétale, avec des

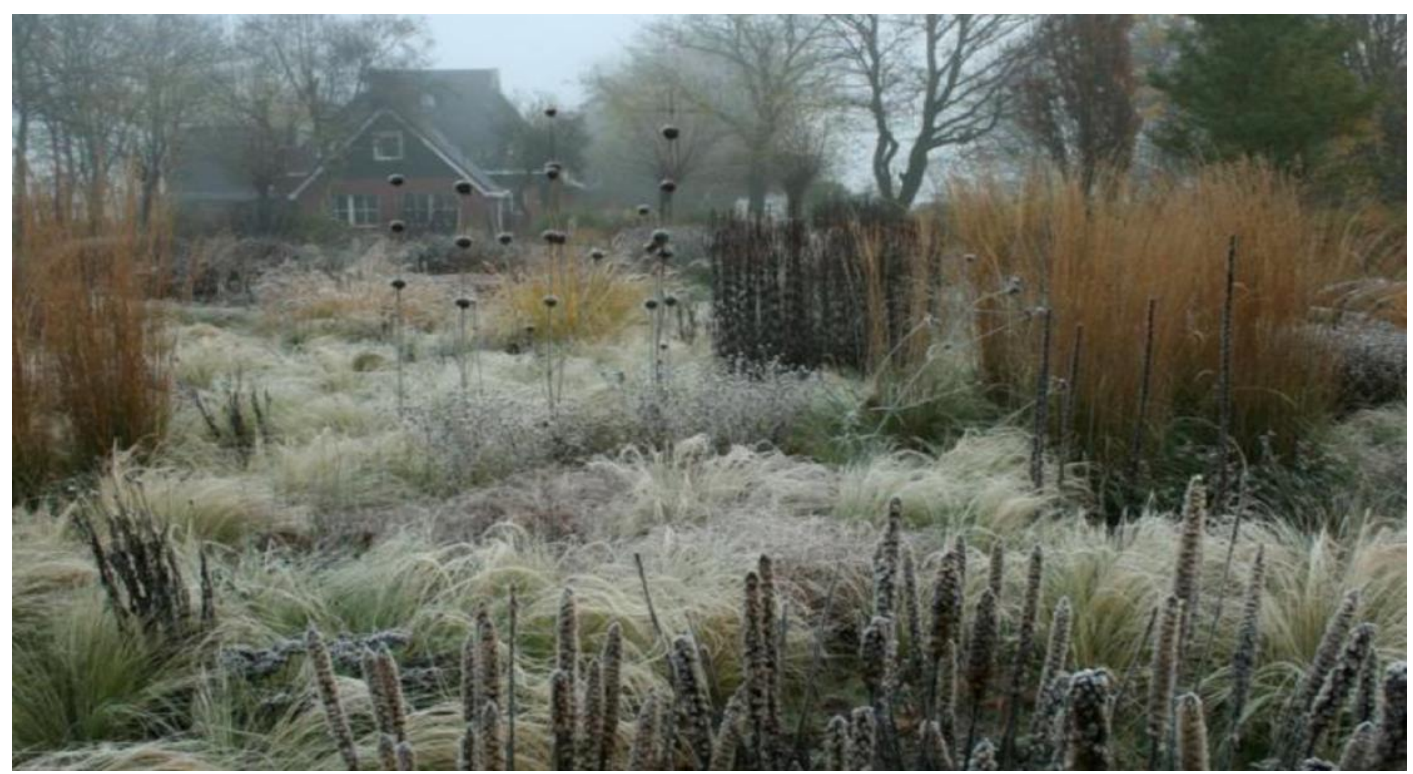

Illustration 31 : Le jardin de Jacostuin, conçu par Jaap de Vries (Pays-Bas)

graminées, chardons, etc.

Dans son «jardin punk $»^{21}$ (illustration 32), Eric Lenoir expérimente le jardinage à partir de sa volonté de faire avec la nature et non contre, en réduisant l'intervention du jardinier au minimum.

Derek Jarman ${ }^{22}$ a créé un jardin en bord de mer (illustration 33), dans un lieu couvert de galets, où il n'y a pas de terre et presque pas de végétation. Tout a commencé avec des silex ramassés sur la plage, puis la replantation d'une aubépine, la protection d'un chou marin, etc. Il ajoute ensuite diverses plantes locales, et utilise autant d'éléments minéraux que végétaux.

\footnotetext{
${ }^{21}$ Lenoir, É. Petit traité du jardin punk.

22 Jarman, D. Un dernier jardin.
} 
Dans la nouvelle approche paysagère qui émerge de ces jardins, on retrouve à la fois des caractéristiques du paysage-système, avec une référence explicite à l'écologie scientifique, du paysage-vivant, avec une attention aux caractéristiques de chaque être qui peuple ce jardin et aux réseaux selon lesquels ils s'organisent, mais aussi du paysage-cosmos, avec une conscience

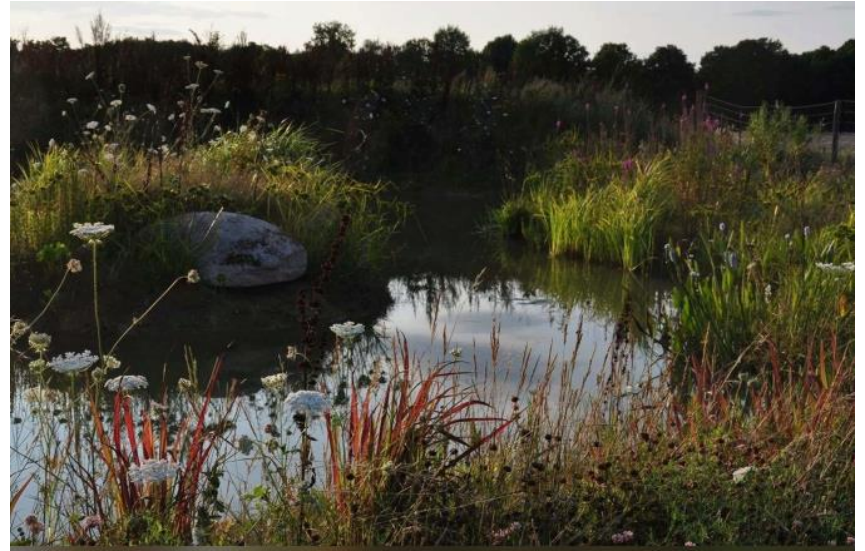

Illustration 32 : pépinière aquatique, dans le jardin expérimental d'Eric Lenoir, Le Flérial (Bourgogne, France)

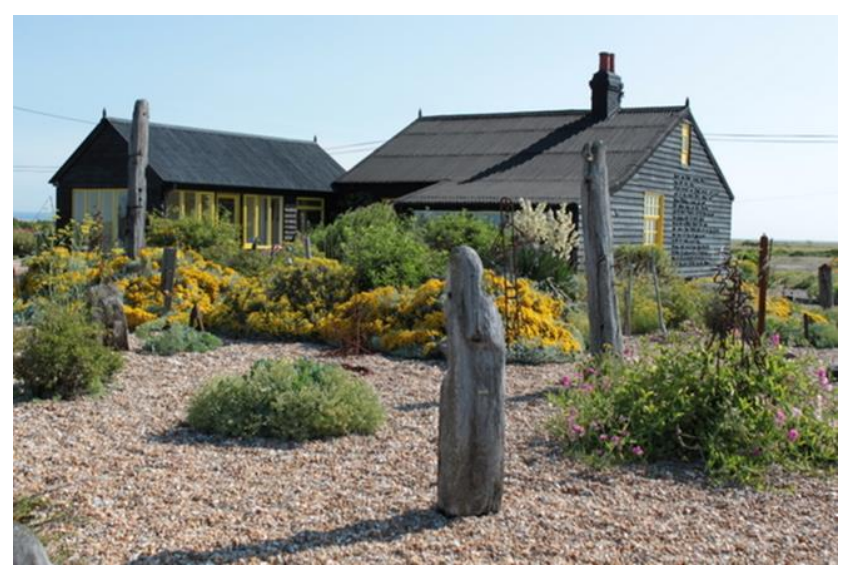

IIlustration 33 : Prospect Cottage, le jardin de bord de mer de Dereck Jarman (Dungeness, Angleterre)

profonde de l'intégration du jardin dans un équilibre écologique à l'échelle planétaire.

Le paysagiste Gilles Clément a ainsi proposé le concept de « jardin planétaire » ${ }^{23}$, pour évoquer la manière dont les activités humaines remodèlent le monde, mais aussi la responsabilité que cela implique par rapport au vivant : il nous faut cultiver le monde comme un jardin vivant, que nous habitons (ce qui différe du modèle associant exploitation et contemplation d'une nature dite sauvage).

Cette perception du monde comme cosmos et cette attention à la manière dont nous l'habitons est également au cœur de la permaculture. La permaculture (illustration 34) est une méthode systémique conçue dans les années soixante-dix par Bill Mollison et David Holmgren ${ }^{24}$, pour concevoir des habitats et des systèmes agricoles, en s'inspirant à la fois de l'écologie scientifique (l'approche écosystémique de Howard Odum), des savoir-faire traditionnels (l'agriculture naturelle de Masanobu Fukuoka) et de principes éthiques (écologistes et humanistes). L'objectif est «la conception consciente de paysages qui miment les modèles et les relations observés dans la nature, visant à obtenir une production abondante de nourriture, de fibres textiles et d'énergie pour satisfaire les besoins locaux. » Cette approche d'une conception écologique par le design a ensuite été élargie à d'autres domaines, comme l'urbanisme ou l'économie.

Ces différents jardins qui combinent les modèles du paysage-système, du paysage-vivant et du paysage-cosmos me semble constituer un nouveau modèle paysager, que je nomme le paysage-milieu de vie.

${ }^{23}$ Clément, G. Le Jardin Planétaire.

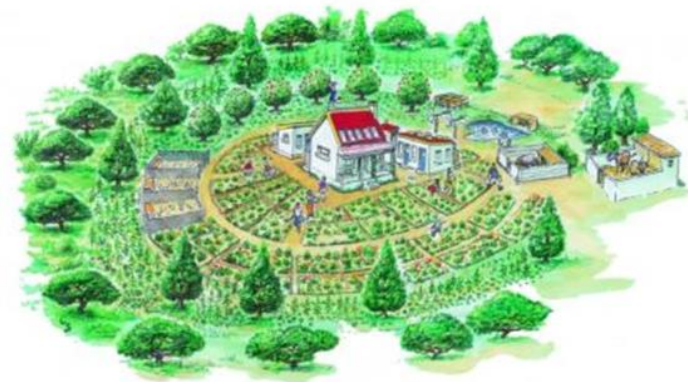

Illustration 34 : modèle schématique d'une ferme en permaculture

${ }^{24}$ Mollison, B. Introduction à la permaculture. 
Pour reconsidérer le paysage à partir de la notion de milieu, je m'inspire de l'approche

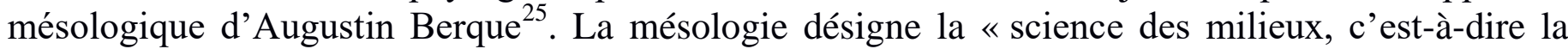
relation spécifique que tout être vivant crée avec son environnement». Elle se distingue selon lui de l'écologie, qui ne se préoccuperait que des caractéristiques physiques et biologique de l'environnement, qui est le même pour tous. Le milieu au contraire est singulier : il est propre à un organisme ou a une espèce, à une personne ou à une société.

Cette approche met donc en avant la subjectivité de la relation établie entre un être vivant et son milieu de vie. Elle est de plus en plus présente dans les pratiques artistiques, depuis les années soixante-dix. Ces artistes expérimentent de nouvelles formes de perception, de présentation et de représentation des paysages, que je nomme écopoétique paysagère.

Cette approche écopoétique des paysages se retrouve d'abord dans des œuvres in situ, réalisées dans et pour un milieu singulier, généralement présentées sous les termes d'art environnemental, de land art ou de d'installations in situ. Au-delà de l'attention au milieu, certaines de ces créations se distinguent par une pratique qui les rapproche également de l'art des jardins : la création avec le vivant, et particulièrement avec le végétal. En voici quelques exemples.

L'artiste herman de vries a réalisé plusieurs Sanctuaires (illustration 35), qui sont des espaces clôts, à l'intérieur desquels il n'y a aucune intervention humaine (la végétation s'y développe librement), avec des ouvertures pour regarder à l'intérieur. Ces « sanctuaires » interrogent le paradoxe de notre relation à la nature. La sculpture de Giuseppe Penone Sentier de charme (illustration 36) est constituée d'une statue en bronze et d'un arbre (un charme bien sûr) entrelacés. La forme de l'œuvre évolue dans le temps selon la croissance de l'arbre. Jean-Philippe Poirée-ville a réalisé une sculpture végétale, formé par une structure sur laquelle poussent librement des mousses,

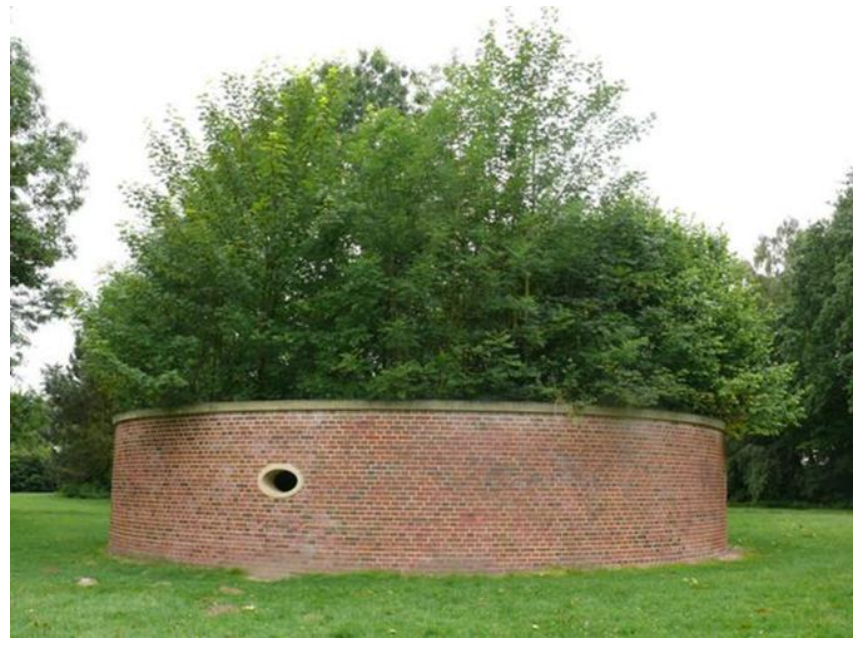

Illustration 35 : Sanctuaire d'herman de vries (1997, Münster, Allemagne)

herbes et fleurs (illustration 37).

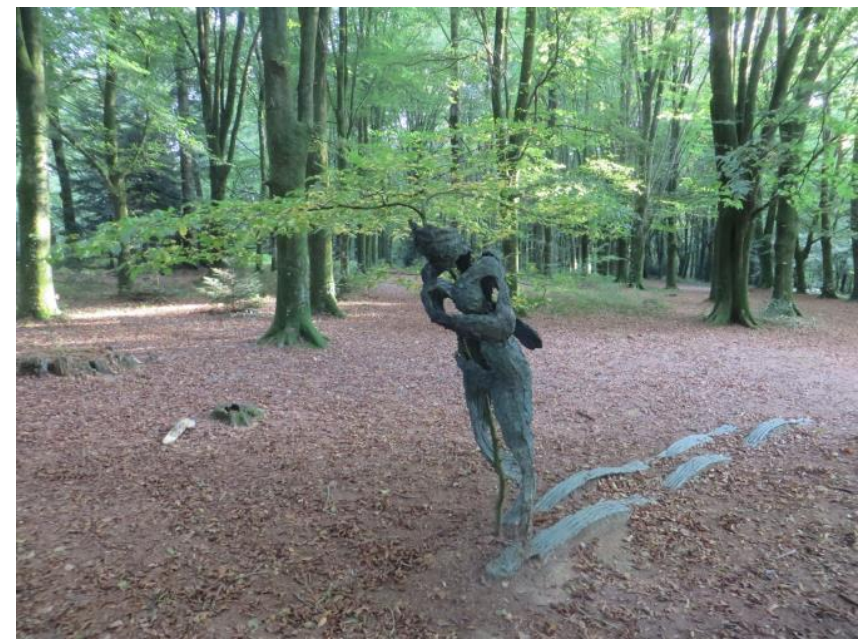

IIlustration 36 : Giuseppe Penone, Sentier de charme (1986, Domaine de Kerguehennec,

\footnotetext{
${ }^{25}$ Berque, A. Là, sur les bords de l'Yvette.
} 


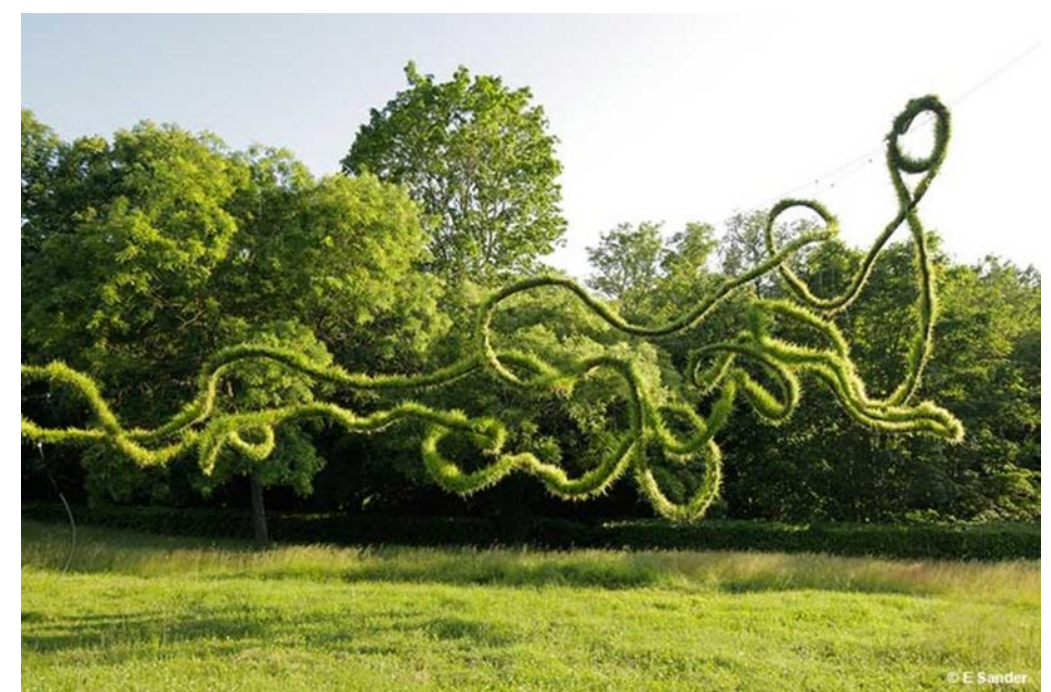

IIlustration 37 : Jean-Philippe Poirée-ville, Sylphe (2012, Domaine de Chaumont-sur-Loire)

Cette approche écopoétique du paysage s'exprime aussi dans des créations in visu, à travers certaines formes de représentation visuelle de lieux existants. Ces artistes utilisent différents médias, notamment la photographie, mais aussi les cartes ou la pratique du relevé, pour raconter l'expérience d'une relation sensible à un milieu.

Paul-Armand Gette adopte une approche de premier abord peu subjective dans son Approche descriptive d'une plage (illustration 38). Il y présente une plage à travers un ensemble de photographies, cartes, diagrammes et mesures climatiques, relevés botaniques et moulages du sol. Cette approche «descriptive » joue avec les codes et méthodes scientifiques, dont cette parodie interroge la prétendue objectivité, en révélant le caractère subjectif et le potentiel poétique de l'expérience du milieu.
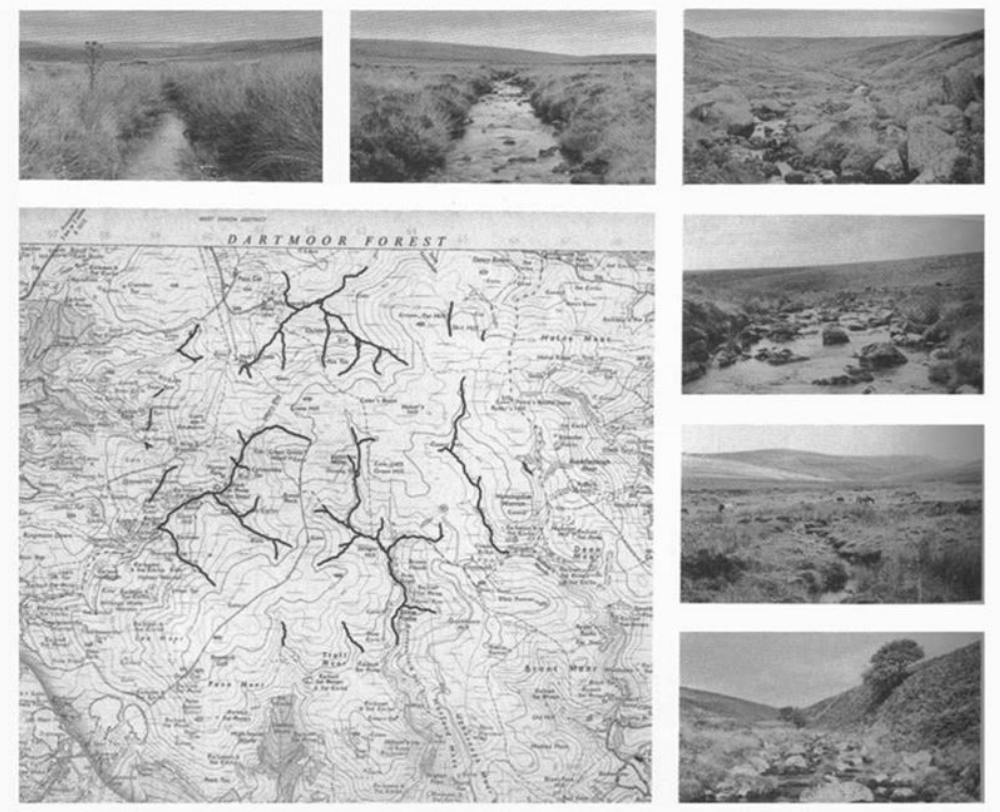

riverbeds (1978, Angleterre)

Illustration 39 : Richard Long, Dartmoor 


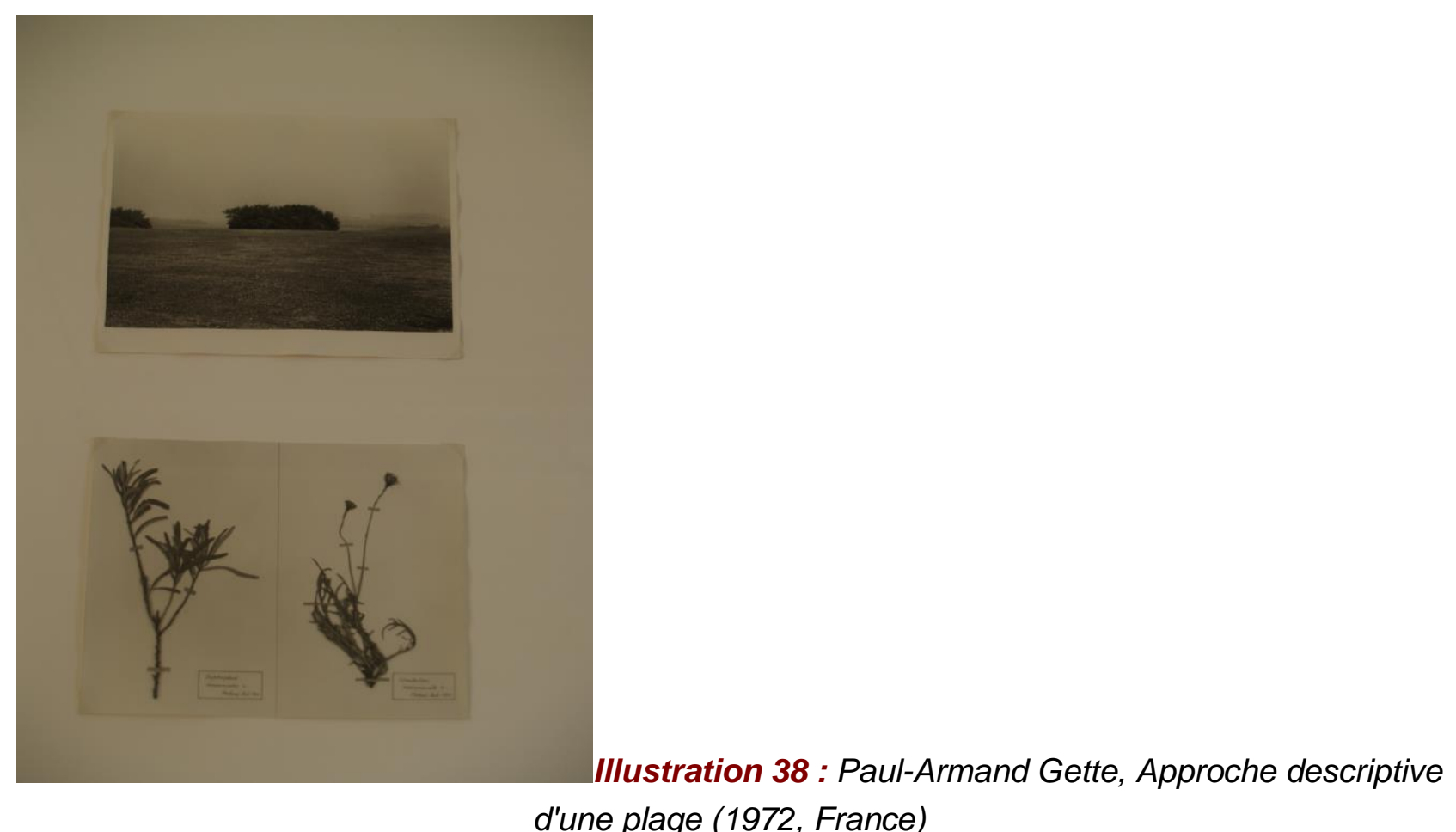
d'une plage (1972, France)

L'œuvre de Richard Long intitulée Dartmoor riverbeds (illustration 39) présente une carte annotée et des photographies retraçant (comme l'indique la légende) « une marche de quatre jours (effectuée par l'artiste) le long de tous les lits de rivières à l'intérieur d'un cercle dans le Dartmoor ». Cette présentation d'apparence documentaire permet ainsi à l'artiste de partager la dimension sensible de son expérience en incitant le spectateur à l'imaginer.

La photographe Lise Gaudaire a enquêté sur l'attachement des paysans à leur terre, à travers la relation sensible qu'ils établissent avec certains milieux. Après des échanges sur la question, elle leur a demandé d'indiquer leur paysage préféré, puis elle a photographié ce paysage ainsi que le paysan concerné. La série photographique Paysans / paysages (illustration 40) associe le portrait de chaque paysan avec la photo de son paysage préféré. Ces photos argentiques sont exposées sous forme de tirage très grand format et publiées aux côtés de ses entretiens avec les paysans photographiés.

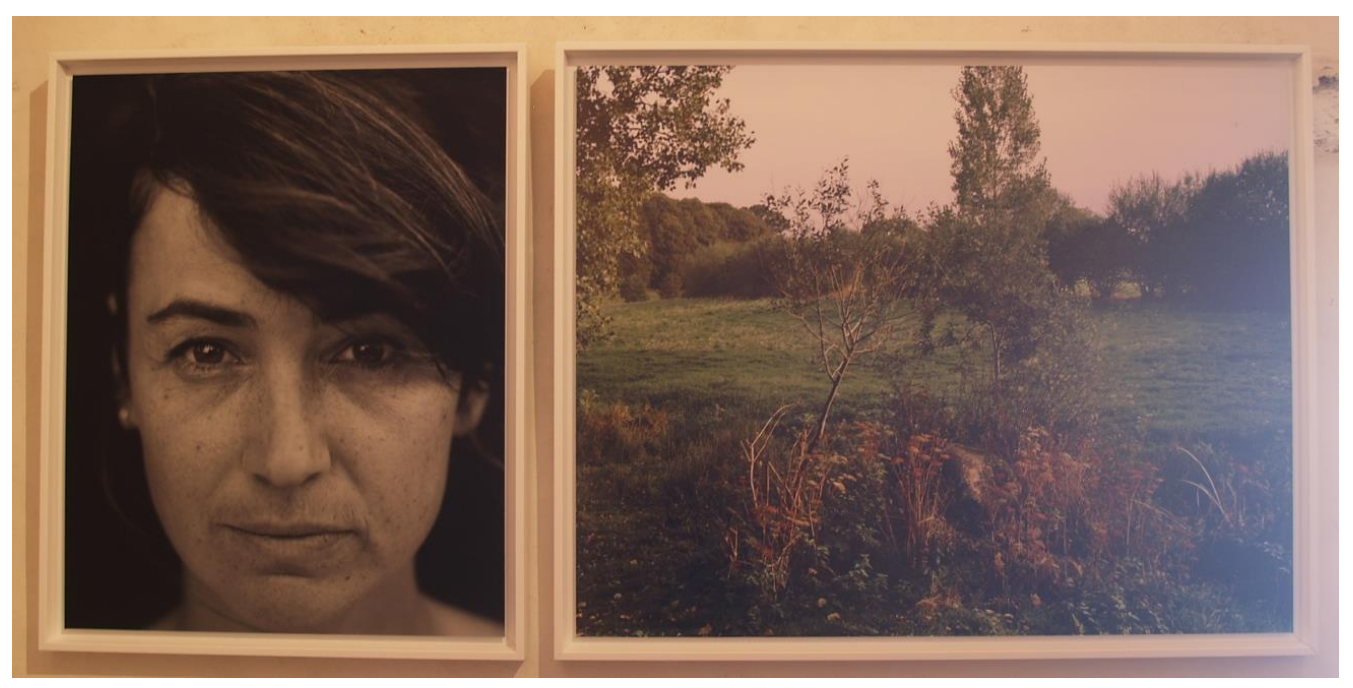

Illustration 40 : Lise Gaudaire, Paysans / paysages (2013-2014, Creuse / Bretagne) 
Ce modèle du paysage-milieu de vie me semble être représentatif d'une approche écopoétique du monde, qui interroge les relations que nous établissons avec notre milieu de vie, et propose des pistes vers des relations plus harmonieuses et non destructrices.

\section{Conclusion. Les enjeux de la fiction verte et de l'écopoétique paysagère}

Pour conclure, je voudrais revenir rapidement sur l'ensemble des modèles paysagers que nous avons traversés. J'espère que ces quelques exemples ont éclaircit la distinction que je propose entre les modèles de la fiction verte, qui sont le paysage-décor, le paysage-émotion, le paysage-spectacle et le paysage-catastrophe ; et les pistes qui s'avancent vers une écopoétique paysagère, celles du paysage-système, du paysage-cosmos, du paysage-vivant et du paysage-milieu de vie.

Au-delà de ces distinctions, on peut s'interroger sur les liens qui peuvent être établis entre ces différents modèles. Par exemple, le paysage-spectacle est une variation ou une évolution du paysage-décor. On remarquera alors que deux de ces modèles sortent du lot. D'un côté, le paysagecatastrophe, dans lequel se retrouvent toutes les caractéristiques du paysage-décor, du paysageémotion et du paysage-spectacle. Il représente donc en quelque sorte le summum de la fiction verte paysagère. De l'autre côté, le paysage-milieu de vie, qui se réfère en même temps au système, au cosmos et au vivant. On peut donc y voir la piste la plus avancée de l'écopoétique paysagère.

On remarque alors que chacun de ces modèles synthétise les caractéristiques de deux approches de l'écologie : l'approche superficielle est exemplifiée dans chaque modèle paysager de la fiction verte, et l'écologie profonde s'exprime dans les modèles écopoétiques. Ce sont alors les enjeux de ces deux approches écologiques du paysage qui sont révélés ici. La fiction verte paysagère, qui aboutit au paysage-catastrophe, révèle que la voie de l'écologie superficielle mène à une impasse. Elle achoppe sur la crise écologique, qu'elle annonce et dénonce, sans trouver d'alternative. En effet, la fiction verte est fondée sur la vision du monde qui est à la source de cette crise. L'écopoétique paysagère, qui se déploie dans le modèle du paysage-milieu de vie, constitue justement cette alternative. L'écopoétique nous ouvre la voie vers une nouvelle vision du monde, qui nous aide à retrouver notre place sur Terre et dans la communauté du Vivant, et donc favorise l'épanouissement de la vie sur Terre.

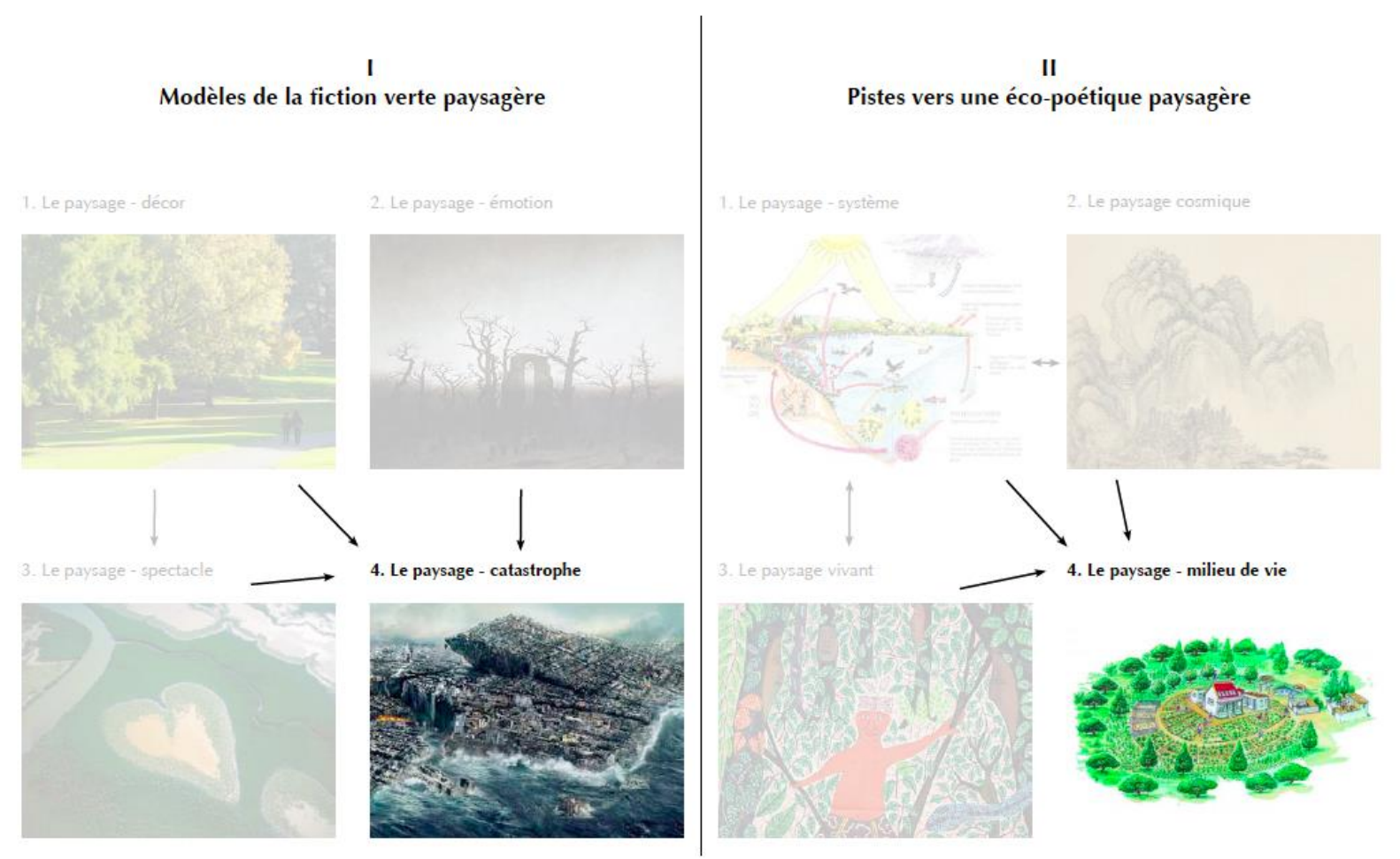




\section{Bibliographie}

Afeissa, Hicham-Stéphane (dir.), Esthétique de l'environnement. Paris, Vrin, 2015, p. 55-84.

Berque, Augustin. Là, sur les bords de l’Yvette: dialogues mésologiques, Éditions Éoliennes, 2017.

Berque, Augustin. Poétique de la terre : histoire naturelle et histoire humaine, essai de mésologie. Paris, Belin, 2014.

Besse, Jean-Marc. Le goût du monde : exercices de paysage. Actes sud / ENSP, Arles / Versailles, 2009.

Cavailles, Roger. «Le philosophe, le paysage et l'écologie», dans Maurand, G. (dir.). Le Paysage : 13e colloque d'Albi Langages et signification. L’Union, C.A.L.S., 1993.

Chelebourg, Christian, Les écofictions : mythologies de la fin du monde. Bruxelles, Les impressions nouvelles, 2012.

Clément, Gilles. Le Jardin planétaire : réconcilier l'homme et la nature. Paris, Parc de la Villette/ Albin Michel, 1999.

Descola, Philippe. Par-delà nature et culture. Paris, Gallimard, 2005.

Descola, Philippe. «Les formes du paysage ». Cours au Collège de France, 2012-2014.

https://www.college-de-france.fr/site/philippe-descola/course-2011-2012.htm

Escande, Yolaine. Montagnes et eaux : la culture du shanshui. Paris, Hermann, 2005.

Fischesser, Bernard et Dupuis-Tate, Marie-France. Le guide illustré de l'écologie. Paris/Versailles, Éditions de La Martinière / Quae, 2007.

Ingold Tim. Marcher avec les dragons. Zones sensibles, Bruxelles, 2013.

Jarman, Derek. Un dernier jardin. Paris, Thames \& Hudson, 2012.

Lenoir, Éric, Petit traité du jardin punk : apprendre à désapprendre. Terre vivante, 2018.

Lovelock, James. La terre est un être vivant : l'hypothèse Gaïa. Paris, Flammarion, 1997.

Mollison, Bill. Introduction à la permaculture, Passerelle Eco, 2012.

Naess, Arne «Le mouvement d'écologie superficielle et le mouvement d'écologie profonde de longue portée. Une présentation ». dans : Afeissa, Hicham-Stéphane (dir.), Éthique de l'environnement. Nature, valeur, respect. Paris, Vrin, 2007. 